COMMUNICATIONS IN

ANALYSIS AND GEOMETRY

Volume 7, Number 1, 1-55, 1999

\title{
Symplectic Topology as the Geometry of Action Functional, II - pants product and cohomological invariants
}

YONG-GEUN OH

\section{Introduction and the main results.}

This is a sequel to our paper [O4] in which we defined the Floer homology of submanifolds $H F_{*}(H, S, J: M)=H F_{*}^{\sigma}(H, S, J: M)$ for given compact submanifold $S \subset M$ and for each given coherent orientation $\sigma \in \operatorname{Or}([S]: M)$, and applied them to the geometry of Lagrangian submanifolds by constructing some homotopy theoretic invariants. Obviously, one can also define the Floer cohomology $H F_{\sigma}^{*}(H, S, J: M)$ by the functorial construction. We will mostly follow the notations we used in [O4]. In this paper we further analyze the case in which $S=M$ and $\sigma$ is the canonical coherent orientation in the sense of Theorem 5.5 [O4] and Theorem 12 [FH1]. Using the Floer theory for this case, we construct some cohomological invariants and study their basic properties in terms of the product structure of Floer cohomology. We refer readers to [O4] for the details of the construction of orientation, grading, and filtration for the Floer (co)homology we use in this paper.

One important new ingredient of our Floer theory in [O4] is the incorporation of some geometric calculations involving the geometry of Lagrangian submanifolds and Hamiltonian systems. Hence our Floer theory can be considered as "geometry of action functional" while Floer's original (global) theory in the literature as "topology of action functional". This optimal calculation has been crucial for applications to problems in symplectic topology, and indeed provides a systematic way of studying symplectic rigidity (See [O6] for a simple proof of non-degeneracy of Hofer's norm in this spirit).

The heart of the present paper is in Section 6 and 7 where we further develop this calculation in a more elaborate way, this time involving pants product and filtrations in Floer cohomology. Our presentation of pants product is given in a way so that an optimal form of this calculation can be carried out, which is crucial for the applications in these sections. In this 
sense, our presentation of the pants product contains new features that have not been addressed in the literature.

In the case $S=M$ from [O4], we have $N^{*} S=o_{M}$. Therefore as far as the global Floer theory is concerned, this case reduces to the standard Floer theory in the dynamical version. Using the symmetry present in this special case,

$$
\begin{aligned}
& \gamma \mapsto \widetilde{\gamma}=\gamma(1-t), \quad u \mapsto \widetilde{u}=u(-\tau, 1-t), \quad \sigma \mapsto \widetilde{\sigma}, \\
& H \mapsto \widetilde{H}=-H(x, 1-t), \quad J \mapsto \widetilde{J}_{t}=J_{1-t}
\end{aligned}
$$

one can define a natural homomorphism between $H F_{n-*}^{\tilde{\sigma}}(\widetilde{H}, \widetilde{J}: M)$ and $H F_{\sigma}^{*}(H, J: M):=H F_{\sigma}^{*}(H, M, J: M)$ which becomes an isomorphism with respect to arbitrary coefficients. This isomorphism enables us to define the semi-infinite version of the cup-product, the so called pants prod$u c t$, on $H F^{*}(H, J: M)$. In general, however, the two Floer homologies $H F_{*}^{\tilde{\sigma}}(\widetilde{H}, \widetilde{J}: M)$ and $H F_{*}^{\sigma}(\widetilde{H}, \widetilde{J}: M)$ are not isomorphic for general coefficients (see Section 2.2 below). We prove in Theorem 2.3 that if we use the canonical coherent orientation defined in Theorem 5.5 [O4], the two become isomorphic with arbitrary coefficients (resp. $\mathbb{Z}_{2}$-coefficients) when $M$ is orientable (resp. non-orientable). With respect to this canonical coherent orientation, combining the above two isomorphisms and the isomorphism

$$
H F_{n-k}^{\sigma}(H, J: M) \rightarrow H F_{n-k}^{\sigma}(\widetilde{H}, \widetilde{J}: M),
$$

we obtain the Poincaré duality isomorphism

$$
P D_{(H, J)}: H F_{n-k}^{\sigma}(H, J: M) \rightarrow H F_{\sigma}^{k}(H, J: M) .
$$

From now on, we will fix the canonical coherent orientation and suppress the $\sigma$ from the notations below in this introduction.

We first recall the group operation on the space of Hamiltonians $H$ : $P \times[0,1] \rightarrow \mathbb{R}$

$$
H \# K(x, t)=H(x, t)+K\left(\left(\phi_{H}^{t}\right)^{-1}(x), t\right) .
$$

We also denote by $\bar{H}$ the inverse of $H$ that is given by the formula

$$
\bar{H}(x, t):=-H\left(\left(\phi_{H}^{t}\right)(x), t\right) .
$$

Our pants product is closely related to this group operation.

Theorem I. Let $H^{\alpha}, H^{\beta}, K^{\alpha}, K^{\beta}$ and $H^{\alpha} \# K^{\alpha}, H^{\beta} \# K^{\beta}$ be generic and $J^{\alpha}, J^{\beta}$ are regular for all three Hamiltonians respectively. 
(1) There exists a natural bi-linear map, denoted by $\cup_{F}$ which is called the pants product

$$
H F^{*}(H, J: M) \otimes H F^{*}(K, J: M) \stackrel{\cup_{F}}{\longrightarrow} H F^{*}(H \# K, J: M)
$$

that respects the filtration induced by values of the corresponding action functional (see [O4]) in the sense that $\cup_{F}$ restricts to

(1.3) $H F_{\left(\lambda_{1}, \infty\right)}^{*}(H, J: M) \otimes H F_{\left(\lambda_{2}, \infty\right)}^{*}(K, J: M)$

$$
\stackrel{\cup_{F}}{\longrightarrow} H F_{\left(\lambda_{1}+\lambda_{2}+\epsilon(K), \infty\right)}^{*}(H \# K, J: M)
$$

where $\epsilon(K)$ is a constant, which vanishes for autonomous Hamiltonians $K$, and it satisfies the inequality

$$
|\epsilon(K)| \leq\|K\|
$$

in general.

(2) The above pants product commutes with the natural isomorphism

$$
h^{\alpha \beta}: H F^{*}\left(H^{\beta}, J^{\beta}: M\right) \rightarrow H F^{*}\left(H^{\alpha}, J^{\alpha}: M\right)
$$

in that the following diagram commutes

$H F^{*}\left(H^{\alpha}, J^{\alpha}: M\right) \otimes H F^{*}\left(K^{\alpha}, J^{\alpha}: M\right) \stackrel{\cup_{F}}{\longrightarrow} H F^{*}\left(H^{\alpha} \# K^{\alpha}, J^{\alpha}: M\right)$

$$
\uparrow h_{H}^{\alpha \beta} \otimes h_{K}^{\alpha \beta} \quad \uparrow h_{H \# K}^{\alpha \beta}
$$

$H F^{*}\left(H^{\beta}, J^{\beta}: M\right) \otimes H F^{*}\left(K^{\beta}, J^{\beta}: M\right) \stackrel{\cup_{F}}{\longrightarrow} H F^{*}\left(H^{\beta} \# K^{\beta}, J^{\beta}: M\right)$.

(3) There exists a natural isomorphism, which we call the (semi-infinite version) of the Thom isomorphism

$$
F_{H}=F_{(H, J)}: H^{*}(M, \mathbb{Z}) \rightarrow H F^{*}(H, J: M)
$$

that commutes with $\cup_{F}$ : the diagram

$$
\begin{array}{ccc}
H F^{*}(H, J: M) \otimes H F^{*}(K, J: M) & \rightarrow & H F^{*}(H \# K, J: M) \\
\uparrow F_{H} \otimes F_{K} & & \uparrow F_{H \# K} \\
H^{*}(M, \mathbb{Z}) \otimes H^{*}(M, \mathbb{Z}) & \vec{u} & H^{*}(M, \mathbb{Z})
\end{array}
$$

commutes. 
(4) There exists an action (which we call the cup action by $u \in H^{*}(M, \mathbb{Z})$ on $H F^{*}(H, J: M) u \mapsto(\cdot) \cup_{F} u$ which satisfies

$$
F_{H}(v) \cup_{F} u=F_{H}(v \cup u)
$$

and which restricts to an action on $H F_{(\lambda, \infty)}^{*}(H, J: M)$ for all $\lambda \in \mathbb{R}$.

There have been several articles (see [BzR], [Sc2] and [PSS]) which describe the pants product as we do in this paper. In fact, they describe more general results in the point of view of the symplectic version of relative Donaldson invariants in the context of Hamiltonian diffeomorphisms. Fukaya [Fu1] also dealt with the Lagrangian intersections in a rather brief way. Floer [F3] first studied a product of the type (1.4) in the cup-length estimate of Lagrangian intersections.

However, for the first time, in this paper the filtration is taken into account as in Theorem I (1) and (4) in the study of pants product. For this purpose, it is crucial to put $H \# K$ on the right hand side of the above diagrams for the pants product $\cup_{F}$ when we restrict to the relative cohomology as in (1.3), although the global version (1.2) will still hold for arbitrary Hamiltonian $L$ not just for $H \# K$. Furthermore, we would like to emphasize that in the proof of the statement (3) (see [FO] for the relevant result), it is again crucial to have $H \# K$ on the right hand side. We call this phenomenon the conservation law for the pants product. This important feature has not appeared so far in the literature partly because almost all of the literatures on the Floer homology deal with Hamiltonian diffeomorphisms not Lagrangian submanifolds. For the Hamiltonian diffeomorphism, there are very few cases for the above filtration to occur or to be useful. These are the cases where the closed symplectic manifold $(P, \omega)$ satisfies $\left.\omega\right|_{\pi_{2}(P)} \equiv 0$. One could also study the exact symplectic manifold with contact type boundary in the spirit of the present paper, which will be a subject of future study.

Unlike Floer's definition [F3] of the action (1.4) which uses a complicated intersection theoretic method, we will define this action using degenerate pants which enables us to prove the commutativity (4) and the statement on the filtration easily from an obvious modification of the analysis in [FO]. This simple definition of the cup action $\cup_{F}$ satisfying (1.4) has been possible due to our previous analytical work done with Fukaya in [FO], where we carefully studied degeneration of the moduli space of marked $J_{g}$-holomorphic discs with Lagrangian boundary conditions of the graphs of $d f_{j}$ 's and proved that this moduli space is diffeomorphic to that of graph flow moduli space of $f_{j}$ 's defined in $[\mathrm{BC}]$ and $[\mathrm{Fu} 1]$. One important point we would like to address is that because we use the functorial definition of the Floer cohomology, we 
never consider taking the Poincaré dual to the cohomology class $u$ in the definition of this action which most literature do and which forces them to mod out the torsion classes in the definition.

Using the Floer's isomorphism, which we call the semi-infinite version of the Thom isomorphism for the fibration $p: \Omega \rightarrow M$, and imitating Viterbo's construction in [V],

$$
F_{(H, J)}: H^{*}(M, \mathbb{Z}) \rightarrow H F^{*}(H, J: M)
$$

we define the real number

$$
\rho(H, u)=\inf _{\lambda}\left\{\lambda \mid j_{\lambda}^{*} F_{(H, J)}(u) \neq 0\right\}
$$

for each $u \in H^{*}(M, \mathbb{Z})$, and prove that it does not depend on $J$, where $j_{\lambda}^{*}: H F^{*} \rightarrow H F_{(-\infty, \lambda]}^{*}$ is the natural homomorphism. We summarize basic properties of $\rho(H, u)$ in the following

Theorem II (Compare with Theorem II [O4]). Let $u \in H^{*}(M, \mathbb{Z})$ and $\rho(H, u)$ as above. Then

(1) All of $\rho(H, u)$ are critical values of $\mathcal{A}_{H}$ on $\Omega$ and satisfies that for two $H^{\alpha}, H^{\beta}$ with $\phi_{H^{\alpha}}^{1}\left(o_{M}\right)=\phi_{H^{\beta}}^{1}\left(o_{M}\right)$, we have

$$
\rho\left(H^{\beta}, u\right)-\rho\left(H^{\alpha}, u\right)=c\left(H^{\alpha}, H^{\beta}\right)
$$

where $c\left(H^{\alpha}, H^{\beta}\right)$ does not depend on $u \in H^{*}(M, \mathbb{Z})$.

(2) When $H \equiv 0, \rho(H, u)=0$ for all $u \in H^{*}(M, \mathbb{Z})$.

(3) For any $u \in H^{*}(M, \mathbb{Z})$,

$$
\int_{0}^{1}-\max \left(H^{\beta}-H^{\alpha}\right) d t \leq \rho\left(H^{\beta}, u\right)-\rho\left(H^{\alpha}, u\right) \leq \int_{0}^{1}-\min \left(H^{\beta}-H^{\alpha}\right) d t .
$$

In particular, combined with (2), we have

$$
\int_{0}^{1}-\max H d t \leq \rho(H, u) \leq \int_{0}^{1}-\min H d t
$$

(4) $\left|\rho\left(H^{\beta}, u\right)-\rho\left(H^{\alpha}, u\right)\right| \leq\left\|H^{\beta}-H^{\alpha}\right\|_{C^{0}}$ and so $H \mapsto \rho(H, u)$ can be extended to a continuous function of $H$ with respect to the $C^{0}$-norm of $H$. 
(5) For any $u, v \in H^{*}(M, \mathbb{Z})$, we have

$$
\rho(H \# K, u \cup v) \geq \rho(H, u)+\rho(K, v)+\epsilon(K)
$$

where $\epsilon(K)$ is the same constant as in (1.3). In particular, one has

$$
\rho(H, u \cup v) \geq \rho(H, u) .
$$

We would like to compare Theorem II with similar results on the Viterbo's invariants $c(L, u)$ defined in [V]: Our invariant has a direct relation to Hofer's geometry while $c(L, u)$ has only indirect one. However, we prove with D. Milinković (see [MO], [Mk] for details) that up to suitable normalizations, our invariants $\rho(L, u)$ coincide with Viterbo's $c(L, u)$. As in [V], one can define a capacity of $L$ as follows

$$
\gamma(L):=\rho\left(H, \mu_{M}\right)-\rho(H, 1) \geq 0
$$

which will not depend on $H$ generating $L$ i.e., such that $L=\phi_{H}^{1}\left(o_{M}\right)$. Now we relate $\gamma(L)$ with Hofer's distance. Define Hofer's distance between Lagrangian submanifolds (Hamiltonian isotopic to each other) by

$$
d\left(L_{1}, L_{2}\right)=\inf _{H: \phi_{H}\left(L_{1}\right)=L_{2}}\|H\| .
$$

The following theorem is the analogue of Corollary $2.3[\mathrm{~V}]$ but with the estimate in relation to Hofer's distance.

Theorem III. (1) $\gamma(L)=0$ if and only if $L=o_{M}$.

(2) $\gamma(L) \leq d\left(L, o_{M}\right)$ and in particular combined with (1), Hofer's distance is nondegenerate.

In Theorem III and [O4], we have introduced two natural capacities (or sizes) of $L, \operatorname{osc}\left(f_{L}\right)$ and $\gamma(L)$ respectively. Here $f_{L}$ is the basic phase function of $L$ defined in [O4]. We have shown by some example in [O4] that

$$
\operatorname{osc}\left(f_{L}\right) \neq \gamma(L)
$$

in general. It seems plausible to us that the inequality

$$
\operatorname{osc}\left(f_{L}\right) \leq \gamma(L)
$$

holds in general. In fact when $L$ is the graph of an exact one-form, we can prove

$$
\operatorname{osc}\left(f_{L}\right)=\gamma(L)=d\left(o_{M}, L\right)
$$

(see [MO] for its proof). This immediately implies 
Corollary. For any smooth function $f$ on $M$, the path

$$
t \mapsto \operatorname{graph}(t d f)
$$

is a (globally) distance minimizing path with respect to Hofer's distance d.

Once we have Theorem II and III, we can apply the above to the compactification of the graphs of Hamiltonian diffeomorphisms $\phi_{H}$ on $\mathbb{R}^{2 n}$. As we mentioned above, another way of studying diffeomorphisms on $\mathbb{R}^{2 n}$ or more generally on any $(P, \omega)$ with $\left.\omega\right|_{\pi_{2}(P)} \equiv 0$ is directly constructing analogues of our invariants for Hamiltonian diffeomorphisms considering their Floer homology, which we will pursue in the future. In a recent preprint "On the action spectrum for closed sympletically aspherical manifolds", M. Schwart carried out this construction for Hamiltonian diffeomorphisms. Combining the construction in the present paper with P. Seidel's result, he, among other things, proved a triangle inequality for the diffeomorphisms which sharpens Theorem II (5) by elliminating the error term $E(K)$ in Theorem II (5) for the case of diffeomorphisms. We will also postpone more elaborate applications of the theory developed in this paper and [O4] to the future works so that this paper does not become too long and its appearance is not delayed by much. We advise that readers who feel uncomfortable about the coherent orientation question safely take $\mathbb{Z}_{2}$-coefficients to follow the main stream of the ideas developed in this paper and refer them to our future work on the orientation problem in the more general context.

The organization of the paper is in order: Section 2 explains Poincaré duality and Floer's isomorphism with orientation in a precise manner. Section 3 provides a functorial construction of pants product which carefully exploits the symmetry mentioned in the beginning. As a result, we prove Theorem I (1) and (2) except the statement on the filtration. Section 4 contains the proof of Theorem I (3). Section 5 provides a new construction, using degenerate pants, of the cup action defined by Floer [F2] and prove Theorem I (4). Section 6 studies interaction of pants product with filtration and Hofer's geometry, and proves Theorem II and all the statements involving filtrations in Theorem I. Section 7 proves Theorem III. In the appendix, we provide a proof of an index formula on a semi-infinite strip which will be needed in the construction of pants product.

We would like to thank K. Fukaya for writing our joint paper [FO] which has greatly reduced the author's burden in this paper of writing up the analytical details concerning the pants product and without which the appearance of this paper would have been much delayed. We also like to thank the unknown referee for numerous critical comments on the previous versions of 
the paper, which have vastly improved the presentation and the English in the present version. This research is supported in part by an NSF grant and a UW Graduate Research Award grant. Part of the results of the present paper were announced and outlined in our survey paper [Section 7, O3] in the Newton proceedings for the program of Symplectic Topology in 1994.

\section{Notations.}

(1) $H \# K(x, t)=H(x, t)+K\left(\left(\phi_{H}^{t}\right)^{-1}(x), t\right)$

(2) $\bar{H}(x, t)=-H\left(\phi_{H}^{t}(x), t\right)$

(3) $\widetilde{H}(x, t)=-H(x, 1-t)$

(4) $\mathcal{H}=\mathcal{H}^{a c}(P)=$ the set of asymptotically constant Hamiltonians on $P$

(5) $\mathcal{H}_{0}=\left\{H \in \mathcal{H} \mid \phi_{H}\left(o_{M}\right) \pitchfork o_{M}\right\}$

(6) $\mathcal{D}_{\omega}^{a c}(P)=$ the set of Hamiltonian diffeomorphisms generated by $\mathcal{H}^{a c}$

(7) $\phi_{H}=$ the time-one map of the equation $\dot{z}=X_{H}(z)$

(8) $H \mapsto \phi$ if and only if $\phi=\phi_{H}$

(9) $o_{M}=$ the zero section of $T^{*} M$

(10) $H \mapsto L$ if and only if $L=\phi_{H}\left(o_{M}\right)$

(11) $z_{H}^{p}:[0,1] \rightarrow T^{*} M ; \quad z_{H}^{p}(t)=\phi_{H}^{t}\left(\left(\phi_{H}^{1}\right)^{-1}(p)\right)$

(12) $\Omega=\left\{z:[0,1] \rightarrow T^{*} M \mid z(0) \in o_{M}\right\}$

(13) $\Omega(M)=\left\{z:[0,1] \rightarrow T^{*} M \mid z(0), z(1) \in o_{M}\right\}$

\section{Conventions.}

(1) The Hamiltonian vector field $X_{H}$ is defined by $\left.X_{H}\right\rfloor \omega=d H$.

(2) An almost complex structure $J$ is said to be compatible to $\omega$, if the bilinear form $\langle\cdot, \cdot\rangle_{J}=\omega(\cdot, J \cdot)$ defines a Riemannian metric. 


\section{Floer cohomology.}

Let $C F(H, M)$ be the set of solutions

$$
\left\{\begin{array}{l}
\dot{z}=X_{H}(z) \\
z(0), z(1) \in o_{M} \subset T^{*} M .
\end{array}\right.
$$

which is the set of critical points of $\mathcal{A}_{H}$ on $\Omega(M)$ (see $C F(H, M: M)$ in $[\mathrm{O} 4])$. This convention will apply to various other objects we have defined in [O4]. Using the grading and the coherent orientation we provided in [O4], we define a $\mathbb{Z}$-module $C F_{k}(H, M)$, for each $k \in \mathbb{Z}$, by the free $\mathbb{Z}$-module over the set of $z \in C F(H, M)$ with

$$
\mu_{M}(z)+\frac{1}{2} \operatorname{dim} M=k .
$$

We will omit the subscript $M$ from $\mu_{M}$ and just denote $\mu=\mu_{M}$.

Let $\sigma$ be the canonical coherent orientation given in Theorem 5.5 [O4]. We form

$$
\begin{gathered}
C F^{k}(H, M):=\operatorname{Hom}\left(C F_{k}(H, M), \mathbb{Z}\right) \\
\delta_{(H, J)}^{\sigma}:=\operatorname{Hom}\left(\partial_{(H, J)}^{\sigma}\right): C F^{k}(H, M) \rightarrow C F^{k+1}(H, M) .
\end{gathered}
$$

Then we define for each $k \in \mathbb{Z}$, the $k$-th Floer cohomology group of $(H, J: M)$ by

$$
H F_{\sigma}^{k}(H, J: M)=\operatorname{Ker} \delta_{(H, J)}^{\sigma} / \operatorname{Im} \delta_{(H, J)}^{\sigma} .
$$

It also follows from the naturality of construction of the cohomology that we have the isomorphism

$$
h^{\alpha \beta}: H F_{\sigma}^{*}\left(H^{\beta}, J^{\beta}: M\right) \rightarrow H F_{\sigma}^{*}\left(H^{\alpha}, J^{\alpha}: M\right)
$$

for two generic $\left(H^{\alpha}, J^{\alpha}\right)$ and $\left(H^{\beta}, J^{\beta}\right)$. This map is induced from the cochain map $h_{\sigma}^{\alpha \beta}: C F^{*}\left(H^{\beta}, J^{\beta}: M\right) \rightarrow C F^{*}\left(H^{\alpha}, J^{\beta}: M\right)$ which is dual to the chain map $h_{\alpha \beta}^{\sigma}: C F_{*}\left(H^{\alpha}, J^{\alpha}: M\right) \rightarrow C F_{*}\left(H^{\beta}, J^{\beta}: M\right.$ ) (see [O4] for more explanation on this map).

\subsection{Thom isomorphism.}

Theorem 5.5 in [O4], applied to the cohomological version of the case $S=M$ immediately gives rise to the natural isomorphism

$$
F_{(H, J)}: H^{*}(M, \mathbb{Z}) \rightarrow H F_{\sigma}^{*}(H, J: M)
$$


that preserves the grading. This isomorphism is natural in the sense that

$$
F_{\left(H^{\alpha}, J^{\alpha}\right)}=h_{\sigma}^{\alpha \beta} \circ F_{\left(H^{\beta}, J^{\beta}\right)}
$$

defined in (2.2). We regard (2.3) as the semi-infinite version of the Thom isomorphism for the fibration $p: \Omega(M) \rightarrow M$. We would like to emphasize that this isomorphism holds with arbitrary coefficients whether or not the manifold $M$ is orientable. Recall that the classical Thom isomorphism requires only that the vector bundle has an orientation, not the base manifold itself. The coherent orientation provided by Theorem 5.2 [O4] can be interpreted as the "orientation" of the fibration $\left.p\right|_{\Omega(M)}: \Omega(M) \rightarrow M$.

\subsection{Poincaré duality.}

We first note that the "semi-infinite fundamental cycle"

$$
\Omega(M)=\left\{\gamma:[0,1] \rightarrow T^{*} M \mid \gamma(0), \gamma(1) \in o_{M}\right\}
$$

has a natural $\mathbb{Z}_{2}$-action that is induced from the time reversal map: $t \mapsto 1-t$. The $\mathbb{Z}_{2}$-action is given by

$$
\gamma \mapsto \widetilde{\gamma}, \quad \widetilde{\gamma}(t):=\gamma(1-t)
$$

This action is compatible with the action on $\mathcal{H}$,

$$
H \mapsto \widetilde{H}, \quad \widetilde{H}(x, t)=-H(x, 1-t)
$$

and also with the action on $\mathcal{J}=$ the set of almost complex structures,

$$
J \mapsto \widetilde{J}, \quad \widetilde{J}(x, t)=J(x, 1-t)
$$

in the following sense: If $z$ is a solution of

$$
\left\{\begin{array}{l}
\dot{z}=X_{H}(z) \\
z(0), z(1) \in o_{M},
\end{array}\right.
$$

then $\widetilde{z} \in C F_{*}(\widetilde{H}: M)$, i.e., $\widetilde{z}$ satisfies

$$
\left\{\begin{array}{l}
\dot{\tilde{z}}=X_{\tilde{H}}(\widetilde{z}) \\
\widetilde{z}(0), \widetilde{z}(1) \in o_{M} .
\end{array}\right.
$$


Furthermore, if $u: \mathbb{R} \times[0,1] \rightarrow T^{*} M$ is a solution of

$$
\left\{\begin{array}{l}
\frac{\partial u}{\partial \tau}+J\left(\frac{\partial u}{\partial t}-X_{H}(u)\right)=0 \\
u(\tau, 0), u(\tau, 1) \in o_{M} \\
\lim _{\tau \rightarrow-\infty} u(\tau)=z^{\alpha}, \lim _{\tau \rightarrow+\infty} u(\tau)=z^{\beta},
\end{array}\right.
$$

then $\widetilde{u}: \mathbb{R} \times[0,1] \rightarrow T^{*} M$ is a solution of

$$
\left\{\begin{array}{l}
\frac{\partial \widetilde{u}}{\partial \tau}+\widetilde{J}\left(\frac{\partial \widetilde{u}}{\partial t}-X_{\tilde{H}}(\widetilde{u})\right)=0 \\
\widetilde{u}(\tau, 0), \widetilde{u}(\tau, 1) \in o_{M} \\
\lim _{\tau \rightarrow-\infty} \widetilde{u}(\tau)=\widetilde{z}^{\beta}, \lim _{\tau \rightarrow+\infty} \widetilde{u}(\tau)=\widetilde{z}^{\alpha},
\end{array}\right.
$$

where

$$
\widetilde{u}(\tau, t):=u(-\tau, 1-t) .
$$

In other words, the map $u \mapsto \widetilde{u}(2.7)$ defines a natural one-to-one correspondence between $\mathcal{M}_{J}\left(H, M: z^{\alpha}, z^{\beta}\right)$ and $\mathcal{M}_{J}\left(\widetilde{H}, M: \widetilde{z}^{\beta}, \widetilde{z}^{\alpha}\right)$. (2.6) should be considered as the "upward" gradient flow of the action functional $\mathcal{A}_{H}$. By (2.1), the assignment

$$
z \mapsto \widetilde{z}
$$

defines a natural isomorphism between $C F_{k}(H, M)$ and $C F_{n-k}(\widetilde{H}, M)$, where $n=\operatorname{dim} M$. This is because we have the following lemma which immediately follows from the definition of the Maslov index (see [RS] and [O4]), and from the identity

$$
\mu(\widetilde{z})+\frac{1}{2} \operatorname{dim} M=-\mu(z)+\frac{1}{2} \operatorname{dim} M=\operatorname{dim} M-\left(\mu(z)+\frac{1}{2} \operatorname{dim} M\right) .
$$

Lemma 2.1. Let $\mu(z)$ be $\mu_{M}(z)$ as defined in Theorem 5.1 [O4] for $S=$ $M$. Then we have

$$
\mu(\widetilde{z})=-\mu(z) .
$$

Using (2.2)-(2.4) and Lemma 2.1, we define the natural homomorphism

$$
\sigma_{H}: C F_{n-k}(\widetilde{H}: M) \rightarrow C F^{k}(H: M)
$$

as follows. For each generator $\widetilde{z} \in C F_{n-k}(\widetilde{H}: M)$ i.e., $z \in \operatorname{Crit} \mathcal{A}_{H}$ with $\mu(z)+\frac{1}{2} n=k$, we define $\sigma_{H}(\widetilde{z}) \in C F^{k}(H: M)=\operatorname{Hom}\left(C F_{k}(H: M), \mathbb{Z}\right)$ by

$$
\begin{aligned}
\sigma_{H}(\widetilde{z})(w) & =1 & & \text { if } w=z \\
& =0 & & \text { otherwise }
\end{aligned}
$$


where $w$ 's are generators of $C F_{k}(H: M)$. We then extend this definition linearly to the whole module $C F_{n-k}(\widetilde{H}: M)$. Now, the map (2.5) naturally push-forwards the coherent orientation $\sigma$ on $\mathcal{M}_{J}\left(H, M: z^{\alpha}, z^{\beta}\right)$ to the orientation $\widetilde{\sigma} \mathcal{M}_{\jmath}\left(\widetilde{H}, M: \widetilde{z}^{\beta}, \widetilde{z}^{\alpha}\right)$. We would like to emphasize that a priori, this orientation $\widetilde{\sigma}$ on $\mathcal{M}_{\mathcal{J}}\left(\widetilde{H}, M: \widetilde{z}^{\beta}, \widetilde{z}^{\alpha}\right)$, is not connected to the one induced there by $\sigma$.

Proposition 2.2. Let $\partial_{(\tilde{H}, \mathcal{J})}^{\tilde{\sigma}}\left(\delta_{(H, J)}^{\sigma}\right)$ be the canonical boundary (coboundary) maps. Then the following diagram

$$
\begin{array}{ccc}
C F_{n-k}(\widetilde{H}: M) & \stackrel{\sigma_{H}}{\longrightarrow} & C F^{k}(H: M) \\
\downarrow \partial_{(\widetilde{J}, \mathcal{J})}^{\tilde{\sigma}} & & \downarrow \delta_{(H, J)}^{\sigma} \\
C F_{n-k-1}(\widetilde{H}: M) & \stackrel{\sigma_{H}}{\longrightarrow} & C F^{(k+1)}(H: M)
\end{array}
$$

commutes. Hence $\sigma_{H}$ induces a chain-isomorphism and so induces a natural isomorphism

$$
\sigma_{(H, J)}: H F_{n-k}^{\tilde{\sigma}}(\widetilde{H}, \widetilde{J}: M) \rightarrow H F_{\sigma}^{k}(H, J: M) .
$$

Proof. It is enough to check

$$
\delta_{(H, J)}^{\sigma} \circ \sigma_{H}(\widetilde{z})=\sigma_{(H, J)} \circ \partial_{(H, J)}^{\tilde{\sigma}}(\widetilde{z})
$$

for any generator $\widetilde{z} \in C F_{n-k}(\widetilde{H}: M)$. To prove this, we compute $\delta_{(H, J)}^{\sigma} \circ$ $\sigma_{H}(\widetilde{z})(w)$ and $\sigma_{(H, J)} \circ \partial_{(\tilde{H}, J)}^{\tilde{\tilde{z}}}(\widetilde{z})(w)$ for each generator $w \in C F_{k+1}(H: M)$. First,

$$
\begin{aligned}
\delta_{(H, J)}^{\sigma} \circ \sigma_{H}(\widetilde{z})(w) & =\sigma_{H}(\widetilde{z})\left(\partial_{(H, J)}^{\sigma} w\right) \\
& =\sigma_{H}(\widetilde{z})\left(\sum_{y} n_{(H, J)}^{\sigma}(w, y) y\right) \\
& =\sum_{y} n_{(H, J)}^{\sigma}(w, y) \delta_{y z} \\
& =n_{(H, J)}^{\sigma}(w, z) .
\end{aligned}
$$


Secondly, we have

$$
\begin{aligned}
\sigma_{H} \circ \partial_{(\widetilde{H}, \widetilde{J})}^{\tilde{\sigma}}(\widetilde{z})(w) & \left.=\sigma_{H}\left(\sum_{\widetilde{y}} n_{(\widetilde{H}, \widetilde{J})}^{\tilde{\sigma}}(\widetilde{z}, \widetilde{y}) \widetilde{y}\right)\right)(w) \\
& =\sum_{\widetilde{y}} n_{(\widetilde{H}, \widetilde{J})}^{\tilde{\sigma}}(\widetilde{z}, \widetilde{y}) \delta_{y w} \\
& =n_{(\widetilde{H}, \Im)}^{\tilde{\sigma}}(\widetilde{z}, \widetilde{w})
\end{aligned}
$$

On the other hand, by the definition of $\tilde{\sigma}$ and $n(\cdot, \cdot)$, we have

$$
n_{(H, J)}^{\sigma}(w, z)=n_{(\widetilde{H}, \mathfrak{J})}^{\tilde{\sigma}}(\widetilde{z}, \widetilde{w})
$$

which finishes the proof.

Remark 2.3. As we pointed out, the two orientations provided by $\sigma$ and $\widetilde{\sigma}$ may not be related in a natural way in general and so the two Floer homology $H F_{n-k}^{\tilde{\sigma}}(\widetilde{H}, \widetilde{J}: M)$ and $H F_{n-k}^{\sigma}(\widetilde{H}, \widetilde{J}: M)$ may not be isomorphic. In fact, when $M$ is connected and not orientable, we know that by the isomorphisms

$$
F_{(H, J)}: H_{*}(M, \mathbb{Z}) \rightarrow H F_{*}^{\sigma}(H, J: M)
$$

and

$$
h_{\alpha \beta}^{\sigma}: H F_{*}^{\sigma}\left(H^{\beta}, J^{\beta}: M\right) \rightarrow H F_{*}^{\sigma}\left(H^{\alpha}, J^{\alpha}: M\right)
$$

we have

$$
H F_{0}^{\sigma}(\widetilde{H}, \widetilde{J}: M) \cong H F_{0}^{\sigma}(H, J: M) \cong H_{0}(M, \mathbb{Z})=\text { free abelian }
$$

while by the isomorphisms (2.2) and (2.8), we have

$$
H F_{0}^{\tilde{\sigma}}(\widetilde{H}, \widetilde{J}: M) \cong H F_{\sigma}^{n}(H, J: M) \cong H^{n}(M, \mathbb{Z})=\text { torsion }
$$

This shows that the Floer cohomologies with respect to different coherent orientations can actually be different.

However, we have the following theorem.

Theorem 2.4. Let $(K, J)$ be any generic parameter. There exists an isomorphism between $H F_{*}^{\sigma}(K, J: M)$ and $H F_{*}^{\tilde{\sigma}}(K, J: M)$ that preserves the grading with $\mathbb{Z}$-coefficients when $M$ is orientable (and so with respect to arbitrary coefficients) and does so with $\mathbb{Z}_{2}$-coefficients when it is non-orientable. 
Proof. We first consider the case when $M$ is orientable. With the natural isomorphism, it will be enough to consider the case when $K=f \circ \pi$ for sufficiently $C^{2}$-small $f$ on $M$ where $\pi: T^{*} M \rightarrow M$ is the canonical projection. Consider the diagram

$$
\begin{array}{ccc}
H F_{n-k}^{\tilde{\sigma}}(K, J: M) & \stackrel{\tau_{K}}{\longrightarrow} & H F_{n-k}^{\sigma}(K, J: M) \\
\uparrow F_{(K, J)} & & \uparrow F_{(K, J)} \\
H_{n-k}^{\tilde{\sigma}}(f, \mathbb{Z}) & \stackrel{\tau_{f}}{\longrightarrow} & H_{n-k}^{\sigma}(f, \mathbb{Z})
\end{array}
$$

where $\tau_{K}: H F_{n-k}^{\tilde{\sigma}}(K, J: M) \rightarrow H F_{n-k}^{\sigma}(K, J: M)$ and $\tau_{f}: H_{n-k}(f, \mathbb{Z}) \rightarrow$ $H^{k}(f, \mathbb{Z})$ are the homomorphisms induced from the identity homomorphism

$$
i d: C F_{n-k}(K, J: M) \rightarrow C F_{n-k}(K, J: M)
$$

and

$$
i d: C_{n-k}(f, \mathbb{Z}) \rightarrow C_{n-k}(f, \mathbb{Z}) .
$$

The definition of the boundary map $\partial_{f}^{\tilde{\sigma}}$ uses the orientation of the Morse complex given by orienting the stable manifolds instead of unstable manifolds, while $\partial_{f}^{\sigma}$ does the one given by the unstable manifolds (see [Section $7, \mathrm{Mi}]$ ). It is also a routine exercise to check that this geometric orientation coincides (maybe upto simultaneous change of sign) with the analytically defined canonical coherent orientation $\sigma$ defined in [FH1, Sc1]. The boundary map $\partial_{K}^{\tilde{\sigma}}$ uses the analogoue to that of $\partial_{f}^{\tilde{\sigma}}$ for the Floer complex.

Note that for $K=f \circ \pi$ sufficiently small and of Morse-Smale type, it has been known (see [Appendix, O5] for the proof) that the kernels of the linearizations of the corresponding trajectories in the Morse complex of $f$ and the Floer complex of $K$ have natural one-to-one correspondence. Therefore we can also naturally identify the two maps

$$
\partial_{K}^{\tilde{\sigma}} \cong \partial_{f}^{\tilde{\sigma}}, \quad \partial_{K}^{\sigma} \cong \partial_{f}^{\sigma} .
$$

When $M$ is orientable, it is proven in Section 7 [Mi] that

$$
\left.\partial_{f}^{\tilde{\sigma}}\right|_{C_{k}(f)}=\left.(-1)^{k-1} \partial_{f}^{\sigma}\right|_{C_{k}(f)}
$$

which proves that

$$
\tau_{f}: H_{n-k}^{\tilde{\sigma}}(f, \mathbb{Z}) \rightarrow H_{n-k}^{\sigma}(f, \mathbb{Z})
$$

becomes an isomorphism. Then by the way how the vertical homomorphisms are defined (see Theorem 5.5 [O4]), the above diagram commutes. 
Furthermore, both vertical arrows are isomorphisms. Therefore the arrow in the top becomes an isomorphism. When $M$ is non-orientable, (2.12) holds $\bmod 2$. So $\tau_{f}$ is an isomorphism in $\mathbb{Z}_{2}$-coefficient.

Remark 2.5. We would like to emphasize that the isomorphism in Theorem 2.4 does not follow from the standard continuity argument as in (2.3). This continuity fails because it is not generally possible to connect the two coherent orientations $\sigma$ and $\widetilde{\sigma}$, as shown in Remark 2.3. The isomorphism in Theorem 2.4 is essentially the classical Poincare duality of the underlying manifold $M$. The (classical) Poincare duality depends on the orientability of the manifold and so holds only for the cohomology with appropriate coefficients, when $M$ is not orientable. For the non-relative Floer theory Theorem 2.4 is always true simply because the symplectic manifold has the canonical orientation. This point has not been addressed carefully in the literature as in [PSS].

Combining (2.10) and Theorem 2.4 applied to $(\widetilde{H}, \widetilde{J})$, we obtain an isomorphism

$$
P D_{(H, J)}: H F_{k}^{\sigma}(H, J: M) \rightarrow H F_{\sigma}^{n-k}(H, J: M)
$$

which is called the Poincaré duality of the Floer theory in literature. However this isomorphism is not natural while the homomorphism (2.10) is so, when one considers the filtration later. In fact, we will use (2.10) in a crucial way in the definition of the pants product but will never use the isomorphism (2.13) in the rest of the paper.

The identity

$$
\mathcal{A}_{\widetilde{H}}(\widetilde{z})=-\mathcal{A}_{H}(z)
$$

and the map defined in (2.5) are important ingredients in the study of filtrations in Section 5 and 6.

\section{Pants product.}

We will fix the canonical coherent orientation $\sigma$ and suppress $\sigma$ from the notations in the rest of the paper. We will take $\mathbb{Z}$ as coefficients unless otherwise specified.

In this section, we study the product properties of $H F^{*}(H, J: M)$ using the so called pants-product. A version of pants-product has been described in several literature (see [BzR], [PSS], [RT] and [Sc2] for example) mostly in 
non-relative Floer theory. Some aspects of the relative point of view were also described in [Fu1,2]. In fact, they described a more general version of the pants-product in the context of the symplectic version of relative Donaldson invariants. Fukaya and the author [FO] studied, degeneration of the Floer moduli space to the Morse moduli space (or graph flow moduli space in the terminology of $[\mathrm{BzC}]$ ) in full analytic details, which is essential to relate the pants products defined in the Morse theory and Floer theory. [FO] also defines and proves the graph flow moduli space in a precise way.

One novelty of our approach to the pants product in this paper is to use the most functorial version for two different reasons:

(1) In applications to the construction of symplectic invariants, it is crucial to analyze how the filtration in the Floer complex is affected under the pants-product, and to estimate the optimal change of the filtrations.

(2) When one tries to relate the the pants-product on $H F^{*}$ and ordinary cup-product on $H^{*}(M, \mathbb{Z})$, the functorial version of the pants-product is essential in some analytical reason (see [FO]).

Both of these two aspects will be crucial in deriving the product inequality (6.1) of the symplectic invariants we will construct in Section 6 .

We first recall how the classical cup product in the cohomology can be defined

$$
H^{*}(M, \mathbb{Z}) \otimes H^{*}(M, \mathbb{Z}) \stackrel{\cup}{\longrightarrow} H^{*}(M, \mathbb{Z})
$$

in the point of view of the Witten's Morse homology (see [Sc1] for a detailed exposition on Morse homology and [BzC] or [Fu1] for the product operation): One chooses a suitable triple of functions $\left(g_{1}, g_{2}, g_{3}\right)$ on $M$ and consider the gradient flows of $g_{i}$ 's, $i=1,2,3$. Floer's construction will define the Morse homology denoted by $H_{*}\left(M: g_{i}\right)$ for $i=1,2,3$ each of them is isomorphic to $H_{*}(M, \mathbb{Z})$ the singular homology of $M$. By the dual construction, one can also define the Morse cohomology $H^{*}\left(M: g_{i}\right)$. Now for a given tree $T$ with 3 edges, we identify (or give coordinates) the edges $e_{1}, e_{2}$ with $[0, \infty)$ (incoming edges) and the edge $e_{3}$ with $(-\infty, 0]$ (outgoing edge). We then consider the map

$$
I: T \rightarrow M
$$

such that the restriction $\chi_{i}=\left.I\right|_{e_{i}}$ to each edge $e_{i}$ satisfies

$$
\left\{\begin{array}{l}
\frac{d \chi_{i}}{d t}=-\operatorname{grad}_{g} g_{i}\left(\chi_{i}\right) \\
\lim _{\tau \rightarrow+\infty} \chi_{i}(\tau)=p_{i} \\
\lim _{\tau \rightarrow-\infty} \chi_{i}(\tau)=p_{3}
\end{array} \quad \text { for } \mathrm{i}=1,2\right.
$$


where $p_{i} \in \operatorname{Crit}\left(g_{i}\right)$. We define by $\mathcal{M}(M: \vec{g}, \vec{p})$ for $\vec{g}=\left(g_{1}, g_{2}, g_{3}\right)$ and

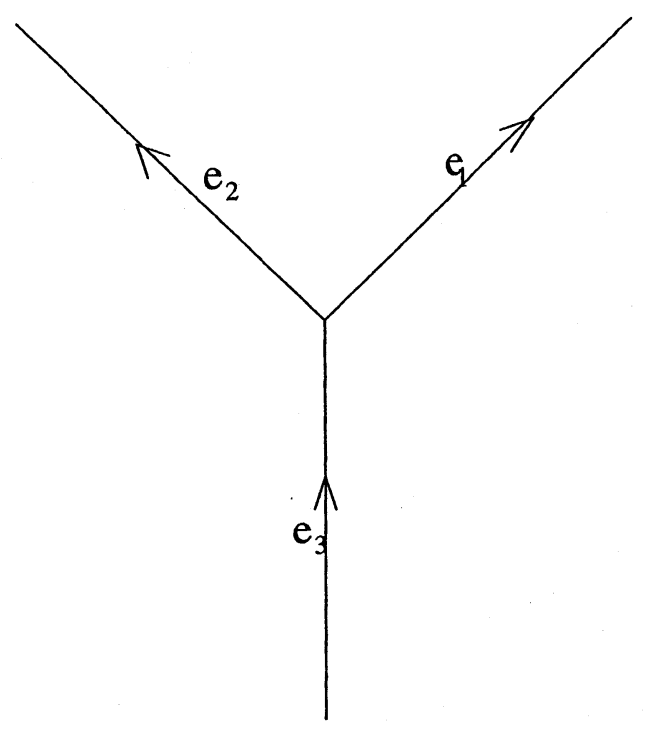

Figure 1

$\vec{p}=\left(p_{1}, p_{2}, p_{3}\right)$ the set of all such maps $I$ as above. Geometrically, one can also identify this set with

$$
\left(\cap_{i=1}^{2} W_{p_{i}}^{+}\left(g_{i}\right)\right) \bigcap W_{p_{3}}^{-}\left(g_{3}\right)
$$

where $W_{p}^{+}(h)$ (resp. $\left.W_{p}^{-}(h)\right)$ is the stable (resp. unstable) manifold of the gradient flow of the function $h$ at the critical point $p \in M$. Under a suitable transversality hypothesis [Theorem 3.1,FO] (see Theorem 4.1 below), the set $\mathcal{M}(M: \vec{g}, \vec{p})$ becomes a smooth manifold of dimension given by

$$
\begin{aligned}
D=D(\vec{p}) & :=\left(n-\mu\left(p_{1}\right)\right)+\left(n-\mu\left(p_{2}\right)\right)+\mu\left(p_{3}\right)+n-3 n, \\
& =\mu\left(p_{3}\right)-\mu\left(p_{1}\right)-\mu\left(p_{2}\right) \\
& =\left(n-\left(n-\mu\left(p_{3}\right)\right)\right)-\mu\left(p_{1}\right)-\mu\left(p_{2}\right)
\end{aligned}
$$

where $\mu\left(p_{j}\right)$ is the Morse index of $g_{j}$ at $p_{j}$. When $D=0$, one can prove for a generic choice of $g_{j}$ 's (see [O2] and [FO] for details) that $\mathcal{M}(M$ : 
$\vec{g}, \vec{p})$ becomes a compact zero-dimensional manifold and so we can count the algebraic number of the elements in $\mathcal{M}(M: \vec{g}, \vec{p})$. We denote this number by $n(M: \vec{g}, \vec{p})$ and define a chain, denoted by $\Psi_{T}(\vec{g}) \in C_{*}\left(M, g_{1}\right) \otimes C_{*}\left(M, g_{2}\right) \otimes$ $C_{*}\left(M,-g_{3}\right)$,

$$
\Psi_{T}(\vec{g}):=\sum_{\vec{p}} n(M: \vec{g}, \vec{p})\langle\vec{p}\rangle,\langle\vec{p}\rangle=p_{1} \otimes p_{2} \otimes p_{3},
$$

where we define $n(M: \vec{g}, \vec{p})=0$ if $D \neq 0$. One can prove by the standard cobordism argument that $\Psi_{T}(\vec{g})$ defines a cycle and so induces an element in $H_{*}\left(M, g_{1}\right) \otimes H_{*}\left(M, g_{2}\right) \otimes H_{*}\left(M,-g_{3}\right)$ which we also denote by $\Psi_{T}(\vec{g})$. Now, we define a bilinear map which we call the pants-product

$$
H^{*}\left(M, g_{1}\right) \otimes H^{*}\left(M, g_{2}\right) \rightarrow H^{*}\left(M, g_{3}\right)
$$

by the dual to the homology class $\Psi_{T}(\vec{g})$. More precisely, for each given $a_{1} \in H^{k}\left(M, g_{1}\right), a_{2} \in H^{\ell}\left(M, g_{2}\right)$, choose their representative cocycles, which we ambiguously denote also by $a_{1}$ and $a_{2}$. Then we consider the contraction cycle

$$
\left\langle a_{1} \otimes a_{2}, \Psi_{T}(\vec{g})\right\rangle \in C_{n-(k+\ell)}\left(M,-g_{3}\right)
$$

and the cocycle

$$
\sigma_{f}\left(\left\langle a_{1} \otimes a_{2}, \Psi_{T}(\vec{g})\right\rangle\right) \in C^{k+\ell}\left(M, g_{3}\right) .
$$

We define the third element $a_{1} \cup a_{2} \in H^{k+\ell}\left(M, g_{3}\right)=H_{\sigma}^{k+\ell}\left(M, g_{3}\right)$ by the cohomology class represented by the cocycle $\sigma_{f}\left(\left\langle a_{1} \otimes a_{2}, \Psi_{T}(\vec{g})\right\rangle\right)$. Here we used the isomorphism $\sigma_{f}$ between $H_{n-(k+\ell)}^{\tilde{\sigma}}\left(M,-g_{3}\right)$ and $H_{\sigma}^{k+\ell}\left(M, g_{3}\right)$ that is defined similarly as (2.8). One can again check by the standard arguments that this class is independent of the choice of the cocycles $a_{1}$ and $a_{2}$. The above construction can be applied to any $\left(g_{1}, g_{2}, g_{3}\right)$ that satisfies suitable transversality hypothesis. However, it was first noted by Fukaya [Fu1] that in relation to the quantized Morse homology, i.e., Floer homology (for the Lagrangian intersections on the cotangent bundle), it is natural to consider the triple $\left(g_{1}, g_{2}, g_{3}\right)$ such that

$$
g_{3}=g_{1}+g_{2}
$$

It turns out that this conservation law is crucial in the analysis needed to establish the equivalence between the Fukaya's $A^{\infty}$ structures in the Morse theory and in its quantization, the Floer theory (see [FO] for detailed proofs). 
The analogue of the conservation law to (3.4) is a crucial ingredient in studying the filtration under the pants-product later in this paper. We recall the operation \# defined in (1.1):

$$
H \# K(x, t)=H(x, t)+K\left(\left(\phi_{H}^{t}\right)^{-1}(x), t\right)
$$

We note that if we apply (1.1) to the time independent Hamiltonians of the form

$$
g \circ \pi: T^{*} M \rightarrow \mathbb{R}
$$

for $g: M \rightarrow \mathbb{R},(1.1)$ reduces to (3.4) because of the following simple formula, which can be easily proved,

$$
(h \circ \pi) \#(k \circ \pi)=(h+k) \circ \pi .
$$

The following is the main theorem we prove throughout the rest of this section, Section 4 and 5. This is the content of Theorem I without taking filtrations into account. We will study its relation to filtrations in Section 6 .

Theorem 3.1. Assume that $H^{\alpha}, H^{\beta}, K^{\alpha}, K^{\beta}$ and $H^{\alpha} \# K^{\alpha}, H^{\beta} \# K^{\beta}$ are in $\mathcal{H}_{0}$ and $J^{\alpha}, J^{\beta}$ are regular with respect to the Hamiltonians respectively of $\alpha$ and $\beta$.

(1) Then there exists a natural bilinear map denoted by $\cup_{F}$ which we call the pants-product and which satisfies the following commutative diagram

$H F^{*}\left(H^{\alpha}, J^{\alpha}: M\right) \otimes H F^{*}\left(K^{\alpha}, J^{\alpha}: M\right) \underset{\mathrm{u}_{F}}{\rightarrow} H F^{*}\left(H^{\alpha} \# K^{\alpha}, J^{\alpha}: M\right)$

$H F^{*}\left(H^{\beta}, J^{\beta}: M\right) \otimes H F^{*}\left(K^{\beta}, J^{\beta}: M\right) \underset{\mathrm{u}_{F}}{\rightarrow} H F^{*}\left(H^{\beta} \# K^{\beta}, J^{\beta}: M\right)$

where the vertical isomorphisms are the ones defined in Theorem 5.4 [O4].

(2) Furthermore $\cup_{F}$ respects the diagram

$$
\begin{array}{ccc}
H F^{*}(H, J: M) \otimes H F^{*}(K, J: M) & \overrightarrow{u_{F}} & H F^{*}(H \# K, J: M) \\
\uparrow F_{H} \otimes F_{K} & & \uparrow F_{H \# K} \\
H^{*}(M, \mathbb{Z}) \otimes H^{*}(M, \mathbb{Z}) & \vec{u} & H^{*}(M, \mathbb{Z}) .
\end{array}
$$


(3) There exists a (right) action by $H^{*}(M, \mathbb{Z})$ on $H F^{*}(H, J: M)$, denoted again by $\cup_{F}$, which respects the diagram

$$
\begin{array}{ccc}
H F^{*}(H, J: M) \otimes H^{*}(M, \mathbb{Z}) & \underset{\cup_{F}}{\longrightarrow} & H F^{*}(H, J: M) \\
\uparrow F_{H} \otimes i d & & \uparrow F_{H} \\
H^{*}(M, \mathbb{Z}) \otimes H^{*}(M, \mathbb{Z}) & \vec{u} & H^{*}(M, \mathbb{Z}) .
\end{array}
$$

Remark 3.2. (1) In the literature, the above product has been described for fixed Hamiltonian or for arbitrary triples without imposing any restriction (in the non-relative Floer theory). [PSS] and [RT] also announced that this product is compatible with the various versions of other products (e.g., the Floer's product [F3] or the quantum cup product).

(2) In Section 6, we will prove a refined version of Theorem 3.1 which takes the filtration into account and use this to prove some product inequality of the symplectic invariants that we will construct.

In the remaining section, we construct the pants-product $\cup_{F}$ and outline the proofs of the statements (1), (2) and (3) in Theorem 3.1.

We will consider the quantized version of the space $\mathcal{M}(M: \vec{g}, \vec{p})$ in the Floer theory. Let $\Theta=\Theta_{0,3}$ be a domain of genus 0 in $\mathbb{C}$ with 3 cylindrical ends. In general, we denote by $\Theta_{g, j}$ the domain in $\mathbb{C}$ with genus $g$ and with $j$ marked points in the boundary. $\bar{\Theta}$ is conformally equivalent to the unit disc with 3 marked points on the boundary $\partial \Theta$. We denote

$$
\begin{aligned}
& \Theta_{i}=\phi_{i}([0, \infty) \times[0,1]) \subset \Theta \text { for } i=1,2 \\
& \left.\Theta_{3}=\phi_{3}((-\infty, 0]) \times[0,1]\right) \subset \Theta
\end{aligned}
$$

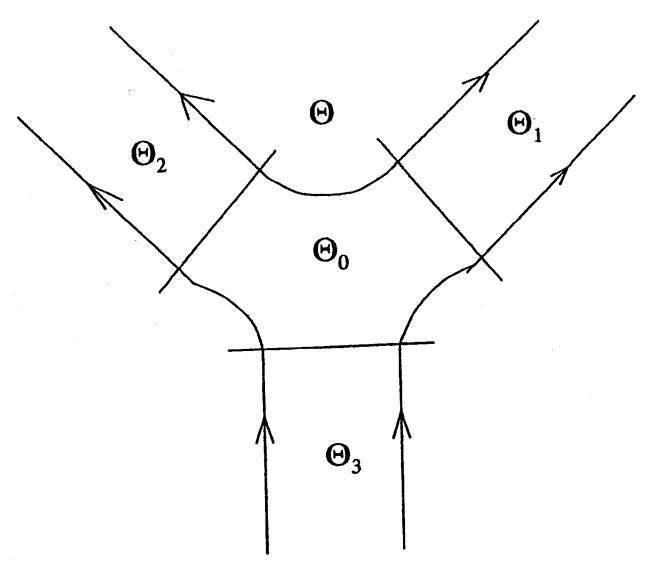


where $\phi_{i}$ 's are one-one holomorphic maps from the corresponding semi-strips into $\mathbb{C}$ respectively. For notational convenience, we also denote

$$
H^{1}=H, H^{2}=K, H^{3}=H \# K, \quad \text { and } \quad \vec{H}=\left(H^{1}, H^{2}, H^{3}\right) .
$$

Given $\vec{z}=\left(z_{1}, z_{2}, z_{3}\right), z_{j} \in C F_{*}\left(H^{j}: M\right)$, we consider the space

$$
\mathcal{M}_{\Theta}(\vec{z})=\mathcal{M}_{\Theta}(\vec{H}, J: \vec{z})
$$

of all smooth maps $u: \Theta \rightarrow T^{*} M$ that satisfy the conditions:

(1) The maps $u_{i}=u \circ \phi_{i}$ satisfy

$$
\left\{\begin{array}{l}
\frac{\partial u}{\partial \tau}+J\left(\frac{\partial u}{\partial t}-X_{H^{i}}(u)\right)=0 \\
\lim _{\tau \rightarrow+\infty} u_{i}(\tau)=z_{i} \\
\lim _{\tau \rightarrow-\infty} u_{3}(\tau)=z_{3}
\end{array} \quad \text { for } \quad i=1,2\right.
$$

(2) u satisfies the boundary condition

$$
u(\partial \Theta) \subset o_{M} \subset T^{*} M
$$

(3) u satisfies "some equation" on the complement

$$
\Theta_{0}=\Theta-\bigcup_{i} \Theta_{i}
$$

that is 0-th order compact perturbation of J-holomorphic equation: Overall on $\Theta$, this defining equation should come from a suitable smooth section of some smooth vector bundle over the space of maps $u: \Theta \rightarrow T^{*} M$ satisfying the boundary condition (2). In (6.7) of Section 6, we will give the precise formula for this equation.

For defining the product, the choice of perturbations over $\Theta_{0}$ is not essential but is essential for studying its relation to filtrations (see Section 6 for the optimal choice). We have the following theorem for the index of $u \in \mathcal{M}_{\Theta}(\vec{H}, J: \vec{z})$. 
Theorem 3.3. For a generic choice of $(\vec{H}, J), \mathcal{M}_{\Theta}(\vec{H}, J: \vec{z})$ becomes an orientable smooth manifold of dimension

$$
\begin{aligned}
D_{(\vec{H}, \vec{J})}(\vec{z}): & =n-\left(\mu\left(z_{1}\right)+\frac{n}{2}\right)-\left(\mu\left(z_{2}\right)+\frac{n}{2}\right)-\left(-\mu\left(z_{3}\right)+\frac{n}{2}\right) \\
& =\left(\mu\left(z_{3}\right)+\frac{n}{2}\right)-\left(\mu\left(z_{1}\right)+\frac{n}{2}\right)-\left(\mu\left(z_{2}\right)+\frac{n}{2}\right)
\end{aligned}
$$

where $n=\operatorname{dim} M$.

Proof. The orientation problem can be solved similarly as in Theorem 5.2 $[\mathrm{O} 4]$ and so we will omit the details except that we would like to emphasize that again our dynamical version of the Floer theory makes it easier to prove that $\mathcal{M}_{\Theta}(\vec{H}, J: \vec{z})$ is orientable. It will be enough to compute the Fredholm index of the linearization operator at $u \in \mathcal{M}_{\Theta}(\vec{H}, J: \vec{z})$. The formula can then be obtained by the gluing formula (or excision formula) by considering the following picture: Cap each $z_{j}$ as drawn in the picture.

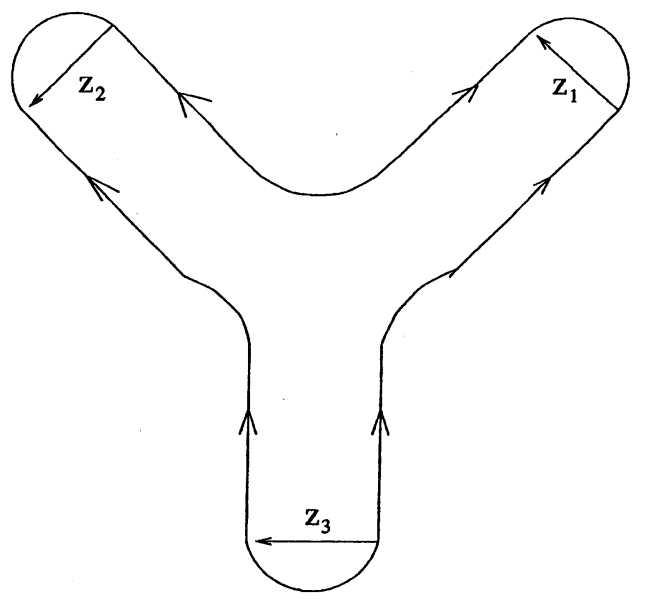

Figure 3

We will prove in the appendix that each cap has the index given by $-\mu(z)+\frac{n}{2}$ when $z$ is an incoming asymptotic limit in the cap. Note that 
the glued operator of the linearized operators over the four regions, under the trivializations of the type satisfying an analogoue to (5.19) in [O4] on $\Theta$, is homotopic to the Cauchy-Riemann operator on the unit disc with boundary condition on $\mathbb{R}^{n} \subset \mathbb{C}^{n}$. It is well-known that the latter operator has the Fredholm index $n$. Now by the excision formula for the Fredholm operator (this can be easily checked by modifying arguments in $[\mathrm{BzR}]$ or [Sc2] that were used for similar applications of the excision formula for the non-relative Floer theory), we have

$$
D_{(\vec{H}, J)}(\vec{z})+\left(\mu\left(z_{1}\right)+\frac{n}{2}\right)+\left(\mu\left(z_{2}\right)+\frac{n}{2}\right)+\left(-\mu\left(z_{3}\right)+\frac{n}{2}\right)=n
$$

which is equivalent to (3.11). This finishes the proof.

Remark 3.4. One can apply the same argument to find the dimension formula for general $\mathcal{M}_{\Theta_{g, k}}\left(\vec{H}, J: \overrightarrow{z_{-}}, \overrightarrow{z_{+}}\right)$, which is the space of solutions of the perturbed Cauchy-Riemann equation that is the generalization of (6.7) for arbitrary $(g, k)$. In fact, for a generic choice of $\vec{H}$, one can prove the following general dimension formula

$\operatorname{dim} \mathcal{M}_{\Theta_{g, k}}\left(\vec{H}, J: \overrightarrow{z_{-}}, \overrightarrow{z_{+}}\right)=n(1-g)-\sum_{j=1}^{k_{-}}\left(-\mu\left(z_{j}^{-}\right)+\frac{n}{2}\right)-\sum_{j=1}^{k_{+}}\left(\mu\left(z_{j}^{+}\right)+\frac{n}{2}\right)$ where $k=k_{-}+k_{+}$, and $k_{-}$and $k_{+}$are the number of outgoing and incoming edges respectively (Similar formula was given in $[\mathrm{BzC}],[\mathrm{BzR}]$ and $[\mathrm{Sc} 2]$ and others in different contexts). Since we do not need this general formula in this paper, we will not discuss more about this generalization.

Furthermore when $D_{(\vec{H}, J)}(\vec{z})=0$, i.e., $\mathcal{M}_{\Theta}(\vec{H}, J: \vec{z})$ has dimension zero, it follows by the standard dimension counting argument that it is compact and so has only finitely many elements, provided we establish the following a priori area estimate. We will prove this in Section 6.

Theorem 3.5. For all $u \in \mathcal{M}_{\Theta}(\vec{H}, J: \vec{z})$ that is defined by the equation (6.7), we have

$\int_{\Theta} \widetilde{u}^{*} \omega=-\mathcal{A}_{H \# K}\left(z_{H \# K}^{p_{3}}\right)+\mathcal{A}_{H \# K}\left(z_{H \# K}^{\phi_{H}\left(p_{1}\right)}\right)-\mathcal{A}_{H}\left(z_{H}^{\phi_{H}\left(p_{1}\right)}\right)+\mathcal{A}_{H}\left(z_{H}^{p_{2}}\right)$

where $\widetilde{u}=\phi_{\vec{z}} \circ u$ is defined in (6.7) below. In particular, we have a priori area bound for $\int_{\Theta} \widetilde{u}^{*} \omega$ that is independent of $u$. 
Then we define an integer

$$
n_{\Theta}(\vec{H}, J: \vec{z})=\#\left(\mathcal{M}_{\Theta}(\vec{H}, J: \vec{z})\right)
$$

and the chain in $C F_{*}(H, J: M) \otimes C F_{*}(K, J: M) \otimes C F_{*}(\widetilde{H \# K}, \widetilde{J}: M)$ by

$$
\Psi_{\Theta}(\vec{H}, J)=\sum n_{\Theta}(\vec{H}, J: \vec{z})\langle\vec{z}\rangle, \quad\langle\vec{z}\rangle=z_{1} \otimes z_{2} \otimes z_{3} .
$$

Again we can prove by the cobordism argument (see $[\mathrm{BzR}]$ ) that this becomes a cycle and so induces a Floer homology class in $H F_{*}(H, J$ : $M) \otimes H F_{*}(K, J: M) \otimes H F_{*}(\widetilde{H \# K}, \widetilde{J}: M)$, which we also denote by $\Psi_{\Theta}(\vec{H}, J)$.

Similarly to the case of Morse cohomology, as in (3.3), for each

$$
\alpha_{1} \in H F^{k}(H, J: M), \alpha_{2} \in H F^{\ell}(K, J: M)
$$

we define

$$
\alpha_{1} \cup_{F} \alpha_{2} \in H F^{k+\ell}(\widetilde{H \# K}, \widetilde{J}: M)
$$

by the cohomology class represented by the dual to the contraction cycle

$$
\left\langle\alpha_{1} \otimes \alpha_{2}, \Psi_{\Theta}(\vec{H}, J)\right\rangle \in C F_{n-(k+\ell)}(\widetilde{H \# K}, \widetilde{J}: M)
$$

i.e., by the cocycle

$$
\sigma_{(H \# K, J)}\left\langle\alpha_{1} \otimes \alpha_{2}, \Psi_{\Theta}(\vec{H}, J)\right\rangle \in C F^{k+\ell}(H \# K, J: M) .
$$

Theorem 3.1 (1) can be proven by now standard arguments considering the boundary of the one-dimensional component of $\mathcal{M}_{\Theta}(\vec{H}, J)$ as in [BzR]. Note that our case is easier because the bubbling does not occur. The existence of an action of $H^{*}(M, \mathbb{Z})$ without the commutative diagram (3.8) was first proven by Floer in [F3] in the context of the geometric version of the Floer theory for Lagrangian intersections. The proofs of Theorem 3.1 (2) and (3) can be reduced to the main result from [FO] (for $k=3$ ). We will explain this reduction in detail in Section 4 and 5 partly because the setting in [FO] is different from that of the present paper and no explicit statement like Theorem 3.1 is given there. Furthermore, our construction in Section 5 using degenerate pants of the cup action (3.8) is new and useful for the proof of commutativity in Theorem 3.1 (3): Our construction can be considered as the limiting case of (3.7) as $\epsilon$ goes to zero for the special Hamiltonian $K$ of the form $F=\epsilon f \circ \pi$ for a suitable Morse function $f$. The commutativity of the diagram (3.8) will follow from the definition of $F_{H}$ and from the result 
in [FO]. This will finish the proof of Theorem I except the statements on the filtration.

The statement about filtration in Theorem I (4) will also immediately follow from this construction.

\section{Proof of Theorem 3.1 (2).}

We would like to briefly recall how one can prove that $H F^{*}(H, J: M)$ is isomorphic to $H^{*}(M, \mathbb{Z})$. A version of this in the Floer's geometric setting $H F_{J}^{*}\left(L_{0}, L_{1}: T^{*} M\right)$

$$
\left\{\begin{array}{l}
\frac{\partial u}{\partial \tau}+J \frac{\partial u}{\partial t}=0 \\
u(\tau, 0) \in o_{M}, u(\tau, 1) \in \phi^{1}\left(o_{M}\right)
\end{array}\right.
$$

was proven by Floer [F2]. Using the invariance property of $H F_{J}^{*}\left(L_{0}, L_{1}\right.$ : $T^{*} M$ ) under the change of $L_{0}, L_{1}$ and $J$, Floer chooses a special choice of $H$ and $J$ to compute the group:

$$
H_{0}=\pi \circ f, \quad J_{H_{0}}=\left(\phi_{H_{0}}^{t}\right)^{*} J_{g}
$$

and considers the map

$$
u \mapsto\left(\phi_{H_{0}}^{t}\right)^{-1} u(\tau, t)=: \widetilde{\chi}(\tau, t)
$$

for each solution $u$ of (4.1). Then he proves that if $|f|_{C^{2}}$ is sufficiently small, the above $\tilde{\chi}$ is $t$-independent and satisfies the equation

$$
\dot{\chi}=-\operatorname{grad}_{g} f(\chi) \text {. }
$$

Conversely, it is easy to check that if $\chi: \mathbb{R} \rightarrow M$ is a solution of (4.3), then

$$
u(\tau, t)=\phi_{H_{0}}^{t}(\chi(\tau))
$$

will be a solution of (4.1) for $J_{H_{0}}$ defined as above. All the solutions of (4.1) will be regular if $f$ is a Morse-Smale function with respect to the given metric $g$ on $M$ and if $|f|_{C^{2}}$ is sufficiently small (See [Appendix, O5]). Therefore by comparing the Morse homology and the Floer homology, we conclude that $H F^{*}\left(H_{0}, J_{H_{0}}: M\right)$ is naturally isomorphic to $H^{*}(M, \mathbb{Z})$. In fact, the above isomorphism holds in the chain level for the choice

$$
H=H_{0} \quad \text { and } \quad J=J_{0}
$$


In the proof of Theorem 3.1 (2), we will follow the similar idea to establish the diffeomorphism between the moduli spaces $\mathcal{M}(M: \vec{g}, \vec{p})$ and $\mathcal{M}_{\Theta}(\vec{H}, J$ : $\vec{z})$ for suitable choice of $(\vec{H}, J)$ and the perturbation of the $J$-holomorphic equation. However unlike the case of maps from the strip $\mathbb{R} \times[0,1]$, it would not be possible to make the corresponding choice $J$ as $J_{H_{0}}$ for the domain $\Theta$, since the definition of $J_{H_{0}}$ in (4.2) involves the coordinates of $\mathbb{R} \times[0,1]$. Therefore it would be important to prove the above equivalence theorem for the canonical almost complex structure $J_{g}$ itself. In [FO], Fukaya and the author analytically constructed a diffeomorphism between $\mathcal{M}(M: \vec{g}, \vec{p})$ and $\mathcal{M}_{\Theta}\left(T^{*} M, J_{g}: \overrightarrow{\Lambda^{\epsilon}}, \overrightarrow{x^{\epsilon}}\right)$ on arbitrary discs with any finite number of marked points by a version of gluing construction. In particular, the main result for $k=2$ proves that there exists a diffeomorphism between the moduli space $\mathcal{M}_{g}(f)$ and the moduli space $\mathcal{M}_{J_{g}}\left(o_{M}\right.$, graph $\left.d f\right)$ when $|f|_{C^{2}}$ is sufficiently small. By comparing the relative indices and orientations, this diffeomorphism gives a chain isomorphism between the Morse complex and the Floer complex.

We state the main theorem for $k=3$ from [FO] for the reader's convenience.

Theorem 4.1 [Theorem 3.1, FO]. Suppose that $f_{i+1}-f_{i}$ are Morse functions and that the unstable manifolds $W_{p}^{-}\left(f_{i+1}-f_{i}\right)$ for $i=$ 1,2,3 (mod 3) intersect transversely, i.e., we have

$$
\prod_{i=1}^{3}\left(W_{p_{i}}^{-}\left(f_{i+1}-f_{i}\right)\right) \pitchfork \Delta \quad \text { in } \quad M \times M \times M
$$

where $\Delta \subset M \times M \times M$ is the diagonal $\Delta=\{(q, q, q) \mid q \in M\}$. Then there exists some $\epsilon_{0}>0$ such that for any $0<\epsilon<\epsilon_{0}$, we have a diffeomorphism

$$
\Phi^{\epsilon}: \mathcal{M}_{g}(M: \vec{g}, \vec{p}) \rightarrow \mathcal{M}_{J_{g}}\left(T^{*} M: \vec{\Lambda}^{\epsilon}, \vec{x}^{\epsilon}\right)
$$

where $g_{i}=f_{i+1}-f_{i}, \vec{\Lambda}^{\epsilon}=\left(\Lambda_{1}^{\epsilon}, \Lambda_{2}^{\epsilon}, \Lambda_{3}^{\epsilon}\right), \quad \vec{x}^{\epsilon}=\left(x_{1}^{\epsilon}, x_{2}^{\epsilon}, x_{3}^{\epsilon}\right)$ and

$$
\Lambda_{i}^{\epsilon}=\operatorname{Graph} \epsilon d f_{i}, \quad x_{i}^{\epsilon}=\left(p_{i}, \epsilon d f_{i}\left(p_{i}\right)\right) .
$$

Note that the three functions $g_{i}=f_{i+1}-f_{i}$ satisfies the conservation law

$$
g_{1}+g_{2}+g_{3}=0
$$

where the sign of change from (3.4) in front of $g_{3}$ is due to the way how [FO] defined the moduli space $\mathcal{M}_{g}(M: \vec{f}, \vec{p})$ : In [FO], we give the coordinates to the tree $T$ so that all the edges are outgoing. 
Now, we would like to establish a similar diffeomorphism between $\mathcal{M}_{g}(M: \vec{g}, \vec{p})$ and our moduli space $\mathcal{M}_{\Theta}\left(\vec{H}, J_{g}: \vec{z}\right)$ where $g_{3}=g_{1}+g_{2}$ and $H^{i}=\pi \circ g^{i}$ and the Hamiltonian path $z_{i}$ is the solution of the equation

$$
\left\{\begin{array}{l}
\dot{z}_{i}=X_{H_{i}}\left(z_{i}\right) \\
z_{i}(1)=p_{i} \in o_{M} \cap \phi_{H}\left(o_{M}\right) \subset T^{*} M \quad \text { for } i=1,2,3
\end{array}\right.
$$

We remind readers of the identity (3.5). By imitating the proof in [FO] for the equation (6.7) which we introduce in Section 6, we can prove the following theorem which is the analogue to Theorem 4.1 in our setting. Since the modification from the proof in [FO] will be straightforward, we omit the details.

Theorem 4.2. Let $J \equiv J_{g}$ and suppose that $g_{1}, g_{2}$ and $g_{3}=g_{1}+g_{2}$ are Morse functions and that the stable manifolds $W_{p_{1}}^{+}\left(g_{1}\right), W_{p_{2}}^{+}\left(g_{2}\right)$ and the unstable manifold $W_{p_{3}}^{-}\left(g_{3}\right)$ intersect transversely. Denote $G_{i}=g_{i} \circ \pi$. Then there exists some $\epsilon_{0}>0$ such that for any $0<\epsilon<\epsilon_{0}$, we have a diffeomorphism

$$
\Psi_{\epsilon}: \mathcal{M}_{g}(M: \vec{g}, \vec{p}) \rightarrow \mathcal{M}_{\Theta}\left(\vec{G}^{\epsilon}, J_{g}: \vec{z}^{\epsilon}\right)
$$

where $\overrightarrow{G^{\epsilon}}=\left(\epsilon G_{1}, \epsilon G_{2}, \epsilon G_{3}\right), \overrightarrow{z^{\epsilon}}=\left(z_{1}^{\epsilon}, z_{2}^{\epsilon}, z_{3}^{\epsilon}\right)$ and $z_{i}^{\epsilon}$ are the solutions of

$$
\left\{\begin{array}{l}
\dot{z}_{i}^{\epsilon}=\dot{X}_{\epsilon G_{i}}\left(z_{i}^{\epsilon}\right) \\
z_{i}(1)=p_{i} \in o_{M}
\end{array}\right.
$$

Furthermore, the images of this map $\Psi_{\epsilon}$ approximate the $\mathcal{M}_{g}(M: \vec{g}, \vec{p})$ in the Hausdorff topology.

Once we have proven Theorem 4.2, the proof of Theorem 3.1 (2) will follow from the definition of the pants-product in the Morse cohomology and our Floor cohomology $H F^{*}(H, J: M)$. The commutativity, Theorem 3.1 (2) (Theorem I (3)) will follow by comparing the boundaries of the onedimensional component of $\mathcal{M}_{\Theta}\left(\vec{G}^{\epsilon}, J_{g}:{\overrightarrow{z^{\epsilon}}}^{\epsilon}\right)$ and $\mathcal{M}_{g}(M: \vec{g}, \vec{p})$. This finally finishes the proof of Theorem 3.1 (2) (Theorem I (3)).

\section{Cup action and the proof of Theorem 3.1 (3).}

Roughly saying, we will define the cup action

$$
H F^{*}(H, J: M) \otimes H^{*}(M, \mathbb{Z}) \rightarrow H F^{*}(H, J: M)
$$


as the dual to a cycle in $H F_{*}(H, J: M) \otimes H_{*}(M, \mathbb{Z}) \otimes H F_{*}(\widetilde{H}, J: M)$. This cycle is defined by counting the elements in $\mathcal{M}_{J}(H, M)$ that intersect with the stable manifolds of critical points of a given Morse function $f$ on $M$. More precisely, for each given $z_{2}, z_{3} \in C F_{*}(H, J: M)$ and $p_{1} \in C_{*}(f: M)$, we consider the following "degenerate pants".

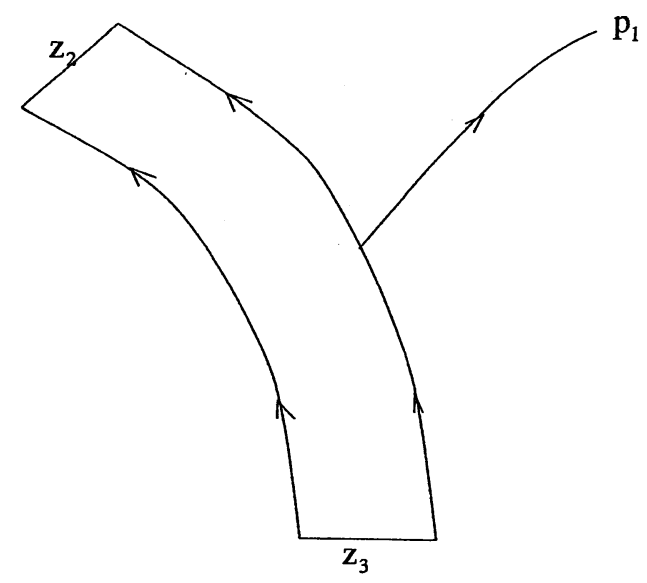

Figure 4

One should regard the current set up as the limit case as $\epsilon \rightarrow 0$ of that in the definition of the pants product in which $K=\epsilon f \circ \pi$. We denote by $\mathcal{M}_{p}^{+}(f)$ the set of gradient trajectories of $f$ that has $p$ as the $\omega$-limit and consider the evaluation map

$$
\mathrm{ev}_{(f: p)}: \mathcal{M}_{f}^{+}(p) \rightarrow M ; \quad e v(\chi)=\chi(0) .
$$

Of course the image of this map is exactly the stable manifold of $p$. Now we define the map

$$
\begin{gathered}
\operatorname{ev}_{\left(f: p_{1}\right)} \times \operatorname{Ev}: \mathcal{M}_{f}^{+}\left(p_{1}\right) \times \mathcal{M}_{J}\left(H, M: z_{3}, z_{2}\right) \rightarrow M \times M \\
(\chi, u) \mapsto(\chi(0), u(0,0))
\end{gathered}
$$

We denote the pre-image $(\mathrm{ev} \times \mathrm{Ev})^{-1}(\Delta)$ by $\mathcal{M}_{J}\left(z_{3}, z_{2} \mid p_{1}\right)=\mathcal{M}_{J}(H$ : $\left.z_{3}, z_{2} \mid f: p_{1}\right)$ where $\Delta \subset M \times M$ is the diagonal. Except the orientation, the following proposition can be proven by the standard transversality argument. The proof of the orientation statement in a much more general 
context will be given elsewhere and so the proof of the following proposition will be omitted. In the mean time, we refer to $[\mathrm{BzR}]$ for some relevant explanation of this orientation question in the non-relative context.

Proposition 5.1. For generic choice of $f$ and $H$, the set $\mathcal{M}_{J}\left(z_{3}, z_{2} \mid p_{1}\right)$ becomes a smooth manifold of dimension given by

$$
\left(\mu\left(z_{3}\right)+\frac{n}{2}\right)-\left(\mu\left(z_{2}\right)+\frac{n}{2}\right)-\mu_{f}\left(p_{1}\right) .
$$

One can also give orientations to these sets which are compatible to the gluing procedure.

Again by a dimension counting argument, one can prove that the zero dimensional component of $\mathcal{M}_{J}(H \mid f)=\cup_{\left(z_{3}, z_{2} \mid p_{1}\right)} \mathcal{M}_{J}\left(z_{3}, z_{2} \mid p_{1}\right)$ will be compact and so we can define an integer

$$
n\left(z_{3}, z_{2} \mid p_{1}\right)=n_{(H, J \mid f)}\left(z_{3}, z_{2} \mid p_{1}\right):=\#\left(\mathcal{M}_{J}\left(z_{3}, z_{2} \mid p_{1}\right)\right) .
$$

We define a chain in $C F_{*}(H, J: M) \otimes C_{*}(f: M) \otimes C F_{*}(\widetilde{H}, \widetilde{J}: M)$ by

$$
\Psi\left(z_{3}, z_{2} \mid p_{1}\right)=\Psi\left(H, J: z_{3}, z_{2} \mid f: p_{1}\right):=\sum_{z_{3}, p_{1} \mid z_{2}} n\left(z_{3}, z_{2} \mid p_{1}\right) z_{2} \otimes p_{1} \otimes \widetilde{z_{3}} .
$$

For each given $a \in C F^{k}(H, J: M)$ and $\beta \in C^{\ell}(f: M)$, we consider the contraction cycle

$$
\left\langle a \otimes \beta, \Psi\left(z_{3}, z_{2} \mid p_{1}\right)\right\rangle=\sum_{z_{3}, z_{2} \mid p_{1}} n\left(z_{3}, z_{2} \mid p_{1}\right) a\left(z_{2}\right) \beta\left(p_{1}\right) \widetilde{z_{3}} \in C F_{n-(k+\ell)}(\widetilde{H}, \widetilde{J}: M)
$$

and then the cocycle

$$
\sigma_{(H, J)}\left\langle a \otimes \beta, \Psi\left(z_{3}, z_{2} \mid p_{1}\right)\right\rangle \in C F^{k+\ell}(H, J: M)
$$

which defines an element in $H F^{k+\ell}(H, J: M)$.

Definition 5.2. For each given $[a] \in H F^{*}(H, J: M)$ and $u \in H^{*}(M, \mathbb{Z})$, we define the cup action of $H^{*}(M, \mathbb{Z})$ on $H F^{*}(H, J: M)$ by

$$
a \cup_{F} u:=\left[\sigma_{(H, J)}\left\langle a \otimes \beta, \Psi\left(z_{3}, z_{2} \mid p_{1}\right)\right\rangle\right]
$$

where $[\beta]=u$. 
It remains to prove the commutativity of the diagram (3.8). By the cobordism argument, it will be enough to prove the case when $H=h \circ \pi$ for a Morse function $h$. The following lemma is the analogoue of Floer's theorem in [F2].

Lemma 5.3. Let $H=h \circ \pi$ where $h$ is a function of Morse-Smale type. Then there exists some $\epsilon>0$ such that if $|h|_{C^{2}}<\epsilon$, all the solutions $u$ with. finite energy of the equation

$$
\left\{\begin{array}{l}
\frac{\partial u}{\partial \tau}+J_{g}\left(\frac{\partial u}{\partial t}-X_{H}(u)\right)=0 \\
u(\tau, 0), u(\tau, 1) \in o_{M}
\end{array}\right.
$$

are $t$-independent and $\chi(\tau)=u(\tau, 0)$ is a trajectory of $-\operatorname{grad} f$.

Now, it is not difficult to see (see $[\mathrm{BzC}]$ for some explanation) that in the Morse-Witten homology setup, the cup action (or product) can be also described as follows: For each $p_{2}, p_{3} \in C_{*}(h: M)$ and $p_{1} \in C_{*}(f: M)$, we study the pair $(\chi, \nu) \in \mathcal{M}_{p_{1}}^{+} \times \mathcal{M}\left(h: p_{3}, p_{2}\right)$ such that

$$
\chi(0)=\nu(0) .
$$

If we define the set of such pairs by $\mathcal{M}\left(h: p_{3}, p_{2} \mid f: p_{1}\right)$, then Lemma 5.3 implies that there exists an orientation preserving diffeomorphism between the space $\mathcal{M}_{J_{g}}\left(H: z_{3}, z_{2} \mid f: p_{1}\right)$ and $\mathcal{M}_{g}\left(h: p_{3}, p_{2} \mid f: p_{1}\right)$, where $z_{3} \equiv \dot{p}_{3}, z_{2} \equiv p_{2}$ are the constant Hamiltonian orbits of $X_{h \circ \pi}$. Then the commutativity (3) immediately follows from the definitions of the cup action. This finishes the proof of Theorem 3.1 (3).

\section{Pants product and cohomological invariants.}

So far, we have proven all the statements in Theorem I except ones involving filtrations in (1).

In this section, we use the (semi-infinite) Thom isomorphism established in Section 2 to construct some cohomological invariants of Viterbo type [V] and prove Theorem II. In the course of doing these, we will also prove Theorem I (1). All the properties of our invariants will be directly related to the Hamiltonian $H$ 's and so to Hofer's geometry.

Using the filtration given by the values of $\mathcal{A}_{H}$ and the canonical grading by $(2.1)$ on $C F_{*}(H, M)$, we define

$$
C F_{a}^{k}=\operatorname{Hom}\left(C F_{k}^{a}, \mathbb{Z}\right)
$$


and

$$
C F_{k}^{a}=C F_{k}^{a}(H, J: M)=\left\{z \in C F_{k}(H, J: M) \mid \mathcal{A}_{H}(z)<a\right\}
$$

as defined in [O4]. Then for $b>a$, we have the homomorphism

$$
j_{b a}^{*}: C F_{b}^{k} \rightarrow C F_{a}^{k}
$$

defined by the restriction, which becomes surjective. Then we define

$$
C F_{(a, b]}^{k}:=\operatorname{Ker} j_{b a}^{*}
$$

The coboundary map $\delta_{(H, J)}: C F^{k}(H: M) \rightarrow C F^{k+1}(H: M)$ induces the homomorphism

$$
\delta_{(H, J)}: C F_{(a, b]}^{k} \rightarrow C F_{(a, b]}^{k+1}
$$

and so one can define the relative Floer cohomology group

$$
H F_{(a, b]}^{k}(H, J: M)=\operatorname{Ker} \delta_{(H, J)} / \operatorname{Im} \delta_{(H, J)} .
$$

It is easy to check from the definition that there exists a canonical homomorphism

$$
j^{*}: H F_{(c, d]}^{k} \rightarrow H F_{(a, b]}^{k}
$$

whenever $c \geq a, d \geq b$. In particular, there exists the natural homomorphism

$$
j_{\lambda}^{*}: H F^{k} \rightarrow H F_{(-\infty, \lambda]}^{k} .
$$

We are now ready to define, for each $u \in H^{*}(M, \mathbb{Z})$, the cohomological invariants. First, we define

$$
\begin{aligned}
\rho(H, J: u) & =\inf \left\{\lambda \mid j_{\lambda}^{*} F_{(H, J)}(u) \neq 0 \text { in } H F_{(-\infty, \lambda]}^{*}(H, J: M)\right\} \\
& =\sup \left\{\lambda \mid j_{\lambda}^{*} F_{(H, J)}(u)=0 \text { in } H F_{(-\infty, \lambda]}^{*}(H, J: M)\right\} .
\end{aligned}
$$

Lemma 6.1. For $(H, J)$ such that $\phi_{H}\left(o_{M}\right) \pitchfork o_{M}$ and $J \in \mathcal{J}_{H}, \rho(H, J: u)$ is a critical value of $\mathcal{A}_{H}$ and independent of $J \in \mathcal{J}_{H}$.

Proof. The proof is an obvious modification of Lemma 7.2 in [O4]. We leave the details to readers.

Definition 6.2. For each $u \neq 0 \in H^{*}(M, \mathbb{Z})$ and $H \in \mathcal{H}_{0}$, we define

$$
\rho(H, u):=\rho(H, J: u)
$$

for some $J \in \mathcal{J}_{H}$ (and so for all $J$ ). We set $\rho(H, 0)=+\infty$ for the zero class $0 \in H^{*}(M, \mathbb{Z})$. 
Now, we study the $H$-dependence of $\rho(H, u)$. The following is again easy to prove as Lemma 8.1 in [O4].

Lemma 6.3. When $H \in \mathcal{H}_{0}$ and as $\|H\|_{C^{1}} \rightarrow 0$, then $\rho(H, u) \rightarrow 0$.

The following theorem summarizes the basic properties of $\rho(H, u)$, which are Theorem II (3)-(5). Note that Theorem II (2) is an immediate consequence of (3).

Theorem 6.4. We assume that $H, H^{\alpha}$ and $H^{\beta} \in \mathcal{H}_{0} \subset \mathcal{H}$. Then

(1) For any $u \neq 0 \in H^{*}(M, \mathbb{Z})$, we have

$$
\int_{0}^{1}-\max _{x}\left(H^{\beta}-H^{\alpha}\right) d t \leq \rho\left(H^{\beta}, u\right)-\rho\left(H^{\alpha}, u\right) \leq \int_{0}^{1}-\min _{x}\left(H^{\beta}-H^{\alpha}\right) d t
$$

In particular when combined with Lemma 6.3, we have

$$
\int_{0}^{1}-\max _{x} H d t \leq \rho(H, u) \leq \int_{0}^{1}-\min H d t .
$$

(2) We have

$$
\left|\rho\left(H^{\beta}, u\right)-\rho\left(H^{\alpha}, u\right)\right| \leq\left\|H^{\beta}-H^{\alpha}\right\|_{C^{0}}
$$

and so the map $H \mapsto \rho(H, u)$ can be extended to a continuous function of $H$ on $\mathcal{H}_{C^{0}}$. We still denote this extension by $\rho(H, u)$ for $u \in H^{*}(M, \mathbb{Z})$.

(3) For any $u, v \in H^{*}(M, \mathbb{Z})$ and $H, K \in \mathcal{H}$, we have

$$
\rho(H \# K, u \cup v) \geq \rho(H, u)+\rho(K, v)+\epsilon(K)
$$

where $\epsilon(K)$ depends only on $K$ which vanishes for any autonomous $K$ and $\epsilon(K) \leq\|K\|$. In particular, we have

$$
\rho(H, u \cup v) \geq \rho(H, u) .
$$

Proof. (2) immediately follows from (1). To prove (1), we follow the argument of the proof of Theorem $7.2(7.2)$ in [O4]. By the same argument as therein, if we set $\epsilon^{\alpha \beta}=\int_{0}^{1}-\min _{x}\left(H^{\beta}-H^{\alpha}\right) d t$, then the natural map (with respect to the "linear homotopy) $h_{\alpha \beta}: C F_{*}\left(H^{\alpha}, J: M\right) \rightarrow C F_{*}\left(H^{\beta}, J: M\right)$ restricts to a map

$$
h_{\alpha \beta}: C F_{*}^{(-\infty, \lambda]}\left(H^{\alpha}, J: M\right) \rightarrow C F_{*}^{\left(-\infty, \lambda+\epsilon^{\alpha \beta}\right]}\left(H^{\beta}, J: M\right)
$$


and so induces the homomorphism

$$
h^{\alpha \beta}: C F_{\left(-\infty, \lambda+\epsilon^{\alpha \beta}\right]}^{*}\left(H^{\beta}, J: M\right) \rightarrow C F_{(-\infty, \lambda]}^{*}\left(H^{\alpha}, J: M\right) .
$$

Therefore for any $\lambda$, we have the following commutative diagram

$$
\begin{array}{cc}
H F^{*}\left(H^{\alpha}, J: M\right) \stackrel{j_{\lambda}^{*}}{\rightarrow} & H F_{(-\infty, \lambda]}^{*}\left(H^{\alpha}, J: M\right) \\
\uparrow h^{\alpha \beta} & \uparrow h^{\alpha \beta} \\
H F^{*}\left(H^{\beta}, J: M\right) \underset{\substack{j_{\lambda+\epsilon^{\alpha \beta}}^{*} \rightarrow \\
\rightarrow}}{H} H F_{\left(-\infty, \lambda+\epsilon^{\alpha \beta}\right]}^{*}\left(H^{\beta}, J: M\right)
\end{array}
$$

where the left hand side arrow is an isomorphism. By naturality of the Floer-Thom isomorphism (2.2) with respect to $h^{\alpha \beta}$ and from the above commutative diagram, for any $u \in H^{*}(M, \mathbb{Z})$, we derive

$$
\rho\left(H^{\beta}, u\right) \leq \rho\left(H^{\alpha}, u\right)+\epsilon^{\alpha \beta},
$$

which finishes the proof of the right half of (1). By changing the role of $\alpha$ and $\beta$ and using the identity

$$
\max f=-\min (-f)
$$

we have finished the proof of the other half of (1). We refer to [O4] for more details in this argument. Now, it remains to prove (3). We first recall the formula from (3.7)

$$
F_{(H \# K)}(u \cup v)=F_{H}(u) \cup_{F} F_{K}(v)
$$

and so one can rewrite $\rho(H \# K, u \cup v)$ as

$$
\begin{aligned}
\rho(H \# K, u \cup v)=\sup _{\lambda}\left\{\lambda \mid j_{\lambda}^{*}\left(F_{H}(u) \cup_{F} F_{K}(v)\right)=0\right. \\
\text { in } \left.H F_{(-\infty, \lambda)}^{*}(H \# K, J: M)\right\}
\end{aligned}
$$

To motivate what we are going to do, we recall how we prove the optimal inequality (8.2) [O4]. We used the linear homotopy

$$
(1-s) H^{\alpha}+s H^{\beta}
$$

connecting $H^{\alpha}$ and $H^{\beta}$. This linear homotopy may not be generic in general but can be approximated by generic paths of Hamiltonians (see [O4]). And all the inequalities we prove are based on the negativity of the kind of term

$$
-\int\left|\frac{\partial u}{\partial t}-X_{H}(u)\right|_{J}^{2} \leq 0
$$


This sort of philosophy is exactly what we need to prove the inequality (6.1).

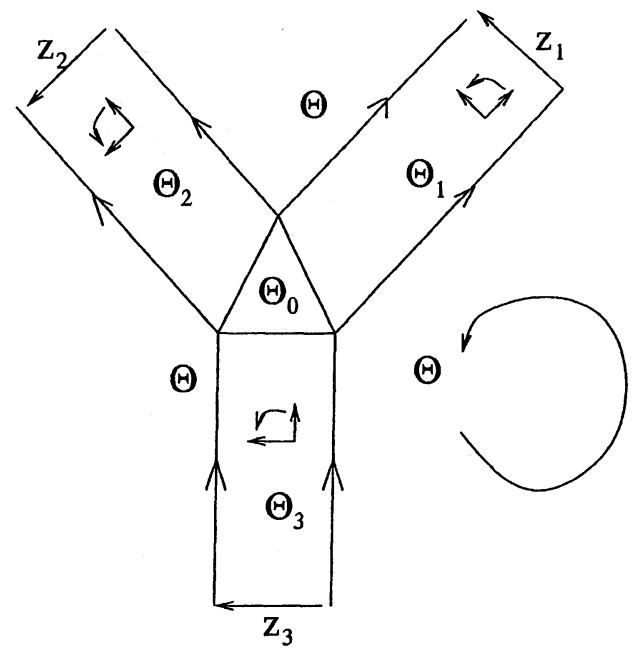

Figure 6

We identify $\Theta$ with the piecewise linear domain drawn as above. We recall that the equation on $\mathbb{R} \times[0,1]$

$$
\left\{\begin{array}{l}
\frac{\partial u}{\partial \tau}+J\left(\frac{\partial u}{\partial t}-X_{H}(u)\right)=0 \\
u(\tau, 0), u(\tau, 1) \in o_{M} \\
u(-\infty)=z^{\alpha}
\end{array}\right.
$$

is equivalent to

$$
\left\{\begin{array}{l}
\frac{\partial \widetilde{u}}{\partial \tau}+J^{H} \frac{\partial \widetilde{u}}{\partial t}=0 \\
\widetilde{u}(\tau, 0) \in \phi_{H}\left(o_{M}\right), \widetilde{u}(\tau, 1) \in o_{M} \\
\widetilde{u}(-\infty)=z^{\alpha}(1) \in \phi_{H}\left(o_{M}\right) \cap o_{M}
\end{array}\right.
$$

where

$$
\begin{aligned}
\widetilde{u}(\tau, t) & :=\phi_{H}^{1}\left(\phi_{H}^{t}\right)^{-1} u(\tau, t) \\
J^{H}(x, t) & :=\left(\phi_{H}^{1}\left(\phi_{H}^{t}\right)^{-1}\right)_{*} J(x, t) .
\end{aligned}
$$

Moreover (6.5) can be re-written as

$$
\bar{\partial}_{J H} \widetilde{u}=0
$$


where

$$
\bar{\partial}_{J^{H}}(\widetilde{u})(z):=\frac{T_{z} \widetilde{u}+J^{H}(\widetilde{u}(z), z) \cdot T \widetilde{u} \circ i}{2}, \quad z=(\tau, t)
$$

An advantage of the equation (6.6) is that it can be written in a coordinatefree form and so can be written on a general domain $\Theta$. Motivated by these discussions, we will choose the perturbed $J$-holomorphic equation needed in the definition of the pants-product defined in Theorem 3.1, in the following way. We first choose a "smooth" map

$$
\phi: \Theta \rightarrow \mathcal{D}_{\omega}^{a c}\left(T^{*} M\right)
$$

that satisfies

(1) $\left.\phi\right|_{\partial_{1} \Theta}=\left(\phi_{H}^{1}\right)^{-1},\left.\phi\right|_{\partial_{2} \Theta}=\phi_{K}^{1}$ and $\left.\phi\right|_{\partial_{3} \Theta}=i d$,

(2) $\left.\phi\right|_{\Theta_{1}}(\tau, t)=\phi_{K}^{1} \circ\left(\phi_{K}^{t}\right)^{-1},\left.\quad \phi\right|_{\Theta_{2}}(\tau, t)=\left(\phi_{H}^{t}\right)^{-1}=\phi_{K}^{1} \circ\left(\phi_{H}^{t} \circ \phi_{K}^{1}\right)^{-1}$, $\left.\phi\right|_{\Theta_{3}}(\tau, t)=\phi_{K}^{1}\left(\phi_{H}^{t} \circ \phi_{K}^{t}\right)^{-1}$

(3) On the center triangle, we define

$$
\phi_{\vec{z}}:=\phi_{K}^{1} \circ\left(\phi_{H}^{t} \circ \phi_{K}^{s}\right)^{-1}
$$

where $\vec{z} \in \Theta_{0}$ is the point pictured as below. Here $\mathcal{D}_{\omega}^{a c}\left(T^{*} M\right)$ is as in the notation (6) in the introduction. It is easy to check that the family of almost complex structures $\left(\phi_{\vec{z}}\right)_{*} J$ naturally extends $J_{H}, J_{K}$ and $J^{H \# K}$ on $\Theta \backslash \Theta_{0}$ to the whole $\Theta$.

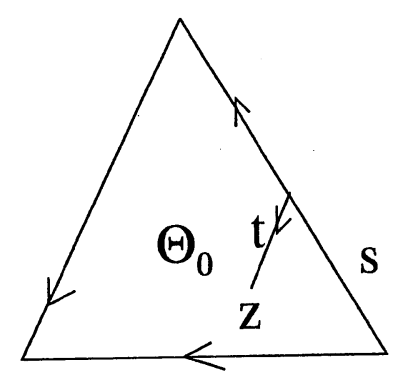

Figure 7 
Now, we impose on $u$ that $u$ satisfy the equation

$$
\left\{\begin{array}{l}
\bar{\partial}_{\left(\phi_{\bar{z}}\right)_{*} J} \widetilde{u}=0, \quad \widetilde{u}=\phi_{\vec{z}} \circ u \\
\widetilde{u}\left(\partial_{1} \Theta\right) \subset\left(\phi_{H}\right)^{-1}\left(o_{M}\right)=\phi_{\bar{H}}\left(o_{M}\right) \\
\widetilde{u}\left(\partial_{2} \Theta\right) \subset \phi_{K}\left(o_{M}\right), \quad \widetilde{u}\left(\partial_{3} \Theta\right) \subset o_{M}
\end{array}\right.
$$

(6.7) is the equation we use in the definition of our pants product given in Section 4. Note that if we restrict (6.7) to each $\Theta_{i}$, it is equivalent to the equation (6.4). Furthermore, we have

$$
\begin{aligned}
& \widetilde{u}_{1}(+\infty)=z_{1}(1) \in \phi_{K}\left(o_{M}\right) \cap o_{M} \\
& \widetilde{u}_{2}(+\infty)=\phi_{H}^{-1}\left(z_{2}(1)\right) \in \phi_{H}^{-1}\left(o_{M}\right) \cap o_{M} \\
& \widetilde{u}_{3}(-\infty)=\phi_{H}^{-1}\left(z_{3}(1)\right) \in \phi_{H}^{-1}\left(o_{M}\right) \cap \phi_{K}\left(o_{M}\right) .
\end{aligned}
$$

all of which are constant paths. We also note that since $\widetilde{u}$ satisfies (6.7), we have

$$
\int_{\Theta} \widetilde{u}^{*} \omega=\int\left|\partial_{\left(\phi_{\vec{z})_{*} J}\right.} \widetilde{u}\right|_{\left(\phi_{\vec{z})_{*} J}\right.}^{2} \geq 0
$$

This is exactly the reason why we choose (6.7) as the required equation in the pants-product. On the other hand, since $\omega=-d \theta$ where $\theta$ is the canonical one form on $T^{*} M$, we have by Stokes' theorem,

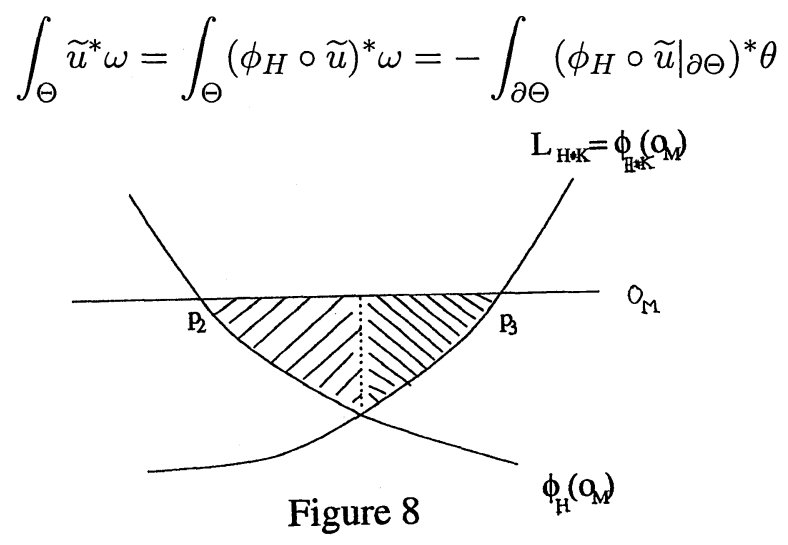

which becomes symplectic area of the shaded region in the picture. Since each $\widetilde{u}\left(\partial_{i} \Theta\right)$ lies in a fixed Lagrangian submanifold, we apply (2.25) in [O4]

$$
d \mathcal{A}_{H}\left(z_{H}^{p}\right)\left(\xi_{H}^{v}\right)=\theta(p)(v)
$$


and re-write the above area (after taking the orientation into consideration) as

$$
\begin{aligned}
\int_{\Theta} \widetilde{u}^{*} \omega= & \mathcal{A}_{H \# K}\left(z_{H \# K}^{p_{3}}\right)-\mathcal{A}_{H \# K}\left(z_{H \# K}^{\phi_{H}\left(p_{1}\right)}\right) \\
& +\mathcal{A}_{H}\left(z_{H}^{\phi_{H}\left(p_{1}\right)}\right)-\mathcal{A}_{H}\left(z_{H}^{p_{2}}\right)
\end{aligned}
$$

This proves Theorem 3.5 which we promised to prove in this section. In fact, the identity (6.9) can be generalized to the domains $\Theta$ with arbitrary number of ends which will imply some generalized version of the inequality (6.2) under the higher order Massey product. Since we will not need this general identity in this paper, we will not discuss further about this generalization.

Now, we further analyze the two middle terms in (6.9).

Lemma 6.5. We have

$$
\mathcal{A}_{H \# K}\left(z_{H \# K}^{\phi_{H}\left(p_{1}\right)}\right)-\mathcal{A}_{H}\left(z_{H}^{\phi_{H}\left(p_{1}\right)}\right)=\mathcal{A}_{K}\left(z_{K}^{p_{1}}\right)+\epsilon\left(K, p_{1}\right)
$$

where

$$
\epsilon\left(K, p_{1}\right)=\int_{0}^{1} \int_{0}^{1} K\left(z_{(1-s) K}^{p_{1}}(t), t\right) d s d t-\int_{0}^{1} K\left(z_{K}^{p_{1}}(t), t\right) d t .
$$

In particular when $K$ is autonomous, we have $\epsilon\left(K, p_{1}\right) \equiv 0$ for all $p_{1} \epsilon$ $\phi_{K}\left(o_{M}\right) \cap o_{M}$.

Assuming this lemma for the moment, we proceed with the proof. Combining (6.8)-(6.10), we have proven that

$$
\mathcal{A}_{H \# K}\left(z_{H \# K}^{p_{3}}\right)-\mathcal{A}_{K}\left(z_{K}^{p_{1}}\right)-\mathcal{A}_{H}\left(z_{H}^{p_{2}}\right)-\epsilon\left(K, p_{1}\right) \geq 0
$$

i.e.

$$
\mathcal{A}_{H \# K}\left(z_{H \# K}^{p_{3}}\right) \geq \mathcal{A}_{K}\left(z_{K}^{p_{1}}\right)+\mathcal{A}_{H}\left(z_{H}^{p_{2}}\right)+\epsilon\left(K, p_{1}\right)
$$

whenever (6.7) has a solution with the asymptotic condition given. We denote

$$
\epsilon(K)=\inf _{p \in \phi_{K}\left(o_{M}\right) \cap o_{M}} \epsilon(K, p) .
$$

Then it follows from the definition

$$
|\epsilon(K)| \leq\|K\|
$$


and that for the autonomous $K$ 's, $\epsilon(K)=0$.

Now, we have to interpret the inequality (6.11) in terms of the wanted inequality (6.1). We consider the diagram (3.7)

$$
\begin{array}{ccc}
H F^{*}(H, J: M) \otimes H F^{*}(K, J: M) & \stackrel{\cup_{F}}{\rightarrow} & H F^{*}(H \# K, J: M) \\
\uparrow F_{H} \otimes F_{K} & & \uparrow F_{H \# K} \\
H^{*}(M, \mathbb{Z}) \otimes H^{*}(M, \mathbb{Z}) & \stackrel{\cup}{\rightarrow} & H^{*}(M, \mathbb{Z})
\end{array}
$$

First if $u \cup v=0$, then $\rho(u \cup v, H)=\infty$ by definition, the inequality is obviously true. Therefore, we assume that $u \cup v \neq 0$ and so $F_{(H, J)}(u \cup v) \neq 0$ since $F_{(H, J)}$ is an isomorphism. To prove (6.1) and Theorem I (1), it will be enough to prove from (6.3) that whenever

$$
\lambda<\rho(H, u)+\rho(K, v)+\epsilon(K),
$$

then

$$
j_{\lambda}^{*}\left(F_{H}(u) \cup_{F} F_{K}(v)\right)=0 .
$$

For such $\lambda$, there exists some $\lambda_{1}$ and $\lambda_{2}$ such that $\lambda_{1}<\rho(H, u), \lambda_{2}<\rho(K, v)$ or $\lambda_{2}<\rho(H, u), \lambda_{1}<\rho(K, v)$, and $\lambda<\lambda_{1}+\lambda_{2}+\epsilon(K)$. We only consider the first case since the other case will be proved in the same way by switching the role of $\lambda_{1}$ and $\lambda_{2}$. By definition of $\rho$, we have

$$
j_{\lambda_{1}}^{*}\left(F_{H}(u)\right)=0 \quad \text { and } \quad j_{\lambda_{2}}^{*}\left(F_{K}(v)\right)=0
$$

Now let $a \in C F^{*}(H, J: M), b \in C F^{*}(K, J: M)$ be the cocycles with $[a]=F_{H}(u),[b]=F_{H}(v)$ respectively and consider the contraction cycle

$$
\left\langle a \otimes b, \Psi_{\Theta}(\vec{H}, J)\right\rangle=\sum n_{\Theta}(\vec{H}, J: \vec{z}) a\left(z_{1}\right) b\left(z_{2}\right) \widetilde{z_{3}} .
$$

(6.12) implies that we can choose the representative cocycles $a$ and $b$ satisfying

$$
a\left(z_{1}\right)=0 \quad \text { and } \quad b\left(z_{2}\right)=0
$$

whenever $\mathcal{A}_{H}\left(z_{1}\right)<\lambda_{1}$ and $\mathcal{A}_{H}\left(z_{2}\right)<\lambda_{2}$ respectively. Therefore only those $z_{1}, z_{2}$ with $\mathcal{A}_{H}\left(z_{1}\right)>\lambda_{1}$ and $\mathcal{A}_{H}\left(z_{2}\right)>\lambda_{2}$ can give non-trivial contribution in (6.13). Furthermore if $n_{\Theta}(\vec{H}, J ; \vec{z}) \neq 0$, then by (6.11)

$$
\begin{aligned}
\mathcal{A}_{H \# K}\left(z_{3}\right) & \geq \mathcal{A}_{K}\left(z_{1}\right)+\mathcal{A}_{H}\left(z_{2}\right)+\epsilon(K) \\
& >\lambda_{1}+\lambda_{2}+\epsilon(K)
\end{aligned}
$$


and so

$$
\mathcal{A}_{H \# K}\left(\widetilde{z_{3}}\right)<-\lambda_{1}-\lambda_{2}-\epsilon(K)
$$

And hence

$$
\left\langle a \otimes b, \Psi_{\Theta}(\vec{H}, J)\right\rangle \in C F_{n-(k+\ell)}^{\left(-\infty,-\lambda_{1}-\lambda_{2}-\epsilon(K)\right)}(\widetilde{H \# K}: M) .
$$

Therefore we have from the definition of $\sigma_{(H, J)}$ that the cocycle

$$
\sigma_{(H \# K, J)}\left\langle a \otimes b, \Psi_{\Theta}(\vec{H}, J)\right\rangle
$$

becomes zero when restricted to

$$
C F_{(k+\ell)}^{\left(-\infty, \lambda_{1}+\lambda_{2}+\epsilon(K)\right]}(H \# K: M)
$$

This implies

$$
j_{\left(\lambda_{1}+\lambda_{2}+\epsilon(K)\right)}^{*}\left(F_{H}(u) \cup F_{H}(v)\right)=0 \quad \text { in } \quad H F_{\left(-\infty, \lambda_{1}+\lambda_{2}+\epsilon(K)\right]}^{(k+\ell)}(H \# K: M) \text {. }
$$

and so $j_{\lambda}^{*}\left(F_{H}(u) \cup F_{H}(v)\right)=0$ since we assumed $\lambda<\lambda_{1}+\lambda_{2}+\epsilon(K)$. This finally finishes the proof of Theorem 6.4 (Theorem II (5)). Note that we have also proven Theorem I (1) as well.

Proof of Lemma 6.5. We connect the Hamiltonians $H \# K$ and $H$ by the path $s \mapsto H^{s}$ by

$$
H^{s}(x, t):=H \#((1-s) K)(x, t)=H(x, t)+(1-s) K\left(\left(\phi_{H}^{t}\right)^{-1}(x), t\right)
$$

and the curves $z_{H \# K}^{\phi_{H}\left(p_{1}\right)}$ and $z_{H}^{\phi_{H}\left(p_{1}\right)}$ by the path $s \mapsto \gamma^{s}$,

$$
\gamma^{s}:=z_{H \#(1-s) K}^{\phi_{H}\left(p_{1}\right)} .
$$

More explicitly, we can write

$$
\begin{aligned}
\gamma^{s}(t) & =\phi_{H}^{t} \circ \phi_{(1-s) K}^{t}\left(( \phi _ { ( 1 - s ) K } ^ { 1 } ) ^ { - 1 } \circ ( \phi _ { H } ^ { 1 } ) ^ { - 1 } \left(\phi_{H}^{1}\left(\left(p_{1}\right)\right)\right.\right. \\
& =\phi_{H}^{t} \circ \phi_{(1-s) K}^{t}\left(\left(\phi_{(1-s) K}^{-1}\left(p_{1}\right)\right) .\right.
\end{aligned}
$$

Note that $\gamma^{s}$ is a Hamiltonian path of $H^{s}$ and

$$
\gamma^{s}(1)=\phi_{H}^{1}\left(p_{1}\right), \quad \gamma^{s}(0)=\left(\phi_{(1-s) K}\right)^{-1}\left(p_{1}\right)=z_{K}^{p_{1}}(s) .
$$

Here the second identity easily follows from the identity

$$
K \# s \bar{K}=(1-s) K
$$


which can be proven by a simple computation. Note that $\gamma^{s}(1)$ is fixed for all $0 \leq s \leq 1$. Now we have

$$
\mathcal{A}_{H}\left(z_{H}^{\phi_{H}\left(p_{1}\right)}\right)-\mathcal{A}_{H \# K}\left(z_{H \# K}^{\phi_{H}\left(p_{1}\right)}\right)=\int_{0}^{1} \frac{d}{d s} \mathcal{A}_{H^{s}}\left(\gamma^{s}\right) d s
$$

where we compute

$$
\frac{d}{d s} \mathcal{A}_{H^{s}}\left(\gamma^{s}\right)=d \mathcal{A}_{H^{s}}\left(\gamma^{s}\right) \frac{\partial \gamma^{s}}{\partial s}+\int_{0}^{1} K\left(\left(\phi_{H}^{t}\right)^{-1}\left(\gamma^{s}(t)\right), t\right)
$$

Applying the general variation formula (2.17) in [O4],

$$
d \mathcal{A}_{H}(\gamma) \xi=\int_{0}^{1}(w(\dot{\gamma}, \xi)-d H(\gamma) \xi)-\langle\xi(0), \theta(\gamma(0))\rangle+\langle\xi(1), \theta(\gamma(1))\rangle
$$

we get

$$
\begin{aligned}
d \mathcal{A}_{H^{s}}\left(\gamma^{s}\right) \frac{\partial \gamma}{\partial s} & =-\left\langle\frac{\partial \gamma^{s}}{\partial s}(0), \theta\left(\gamma^{s}(0)\right)\right\rangle=-\left\langle\dot{z}_{K}^{p_{1}}, \theta\left(z_{K}^{p_{1}}\right)\right\rangle(s) \\
& =-\left(z_{K}^{p_{1}}\right)^{*} \theta(s) .
\end{aligned}
$$

And

$$
\begin{aligned}
\left.\int_{0}^{1} K\left(\phi_{H}^{t}\right)^{-1} \gamma^{s}(t), t\right) d t & =\int_{0}^{1} K\left(\phi_{(1-s)}^{t}\left(\phi_{(1-s) K}^{1}\right)^{-1}(p), t\right) d t \\
& =\int_{0}^{1} K\left(z_{(1-s) K}^{p_{1}}(t), t\right) d t
\end{aligned}
$$

Therefore, we get by integrating (6.15)

$$
\begin{aligned}
\int_{0}^{1} \frac{d}{d s} \mathcal{A}_{H^{s}}\left(\gamma^{s}\right) d s & =-\int_{0}^{1}\left(z_{K}^{p_{1}}\right)^{*} \theta(s)+\int_{0}^{1} \int_{0}^{1} K\left(z_{(1-s) K}^{p_{1}}(t), t\right) d t d s \\
& =-\mathcal{A}_{K}\left(z_{K}^{p_{1}}\right)+\epsilon\left(K, p_{1}\right)
\end{aligned}
$$

where for each $p \in \phi_{K}\left(o_{M}\right) \cap o_{M}, \epsilon(K, p)$ is defined as

$$
\epsilon(K, p)=\int_{0}^{1} \int_{0}^{1} K\left(z_{(1-s) K}^{p}(t), t\right) d s d t-\int_{0}^{1} K\left(z_{K}^{p}(t), t\right) d t .
$$

Now substituting this into (6.14), we have finished the proof of Lemma 6.5. 


\section{Non-triviality of the invariants.}

Note that an immediate consequence of (6.2) applied to $u=1$ and $v=\mu_{M}$ is

$$
\rho\left(H, \mu_{M}\right) \geq \rho(H, 1) .
$$

Now as in [V], we define a capacity of $L$ as follows, which is the analogoue of Viterbo's in [V].

Definition 7.1 [ $\rho$-capacity]. For any $L$ that is Hamiltonian isotopic to $o_{M}$ i.e., $L=\phi\left(o_{M}\right)$ for $\phi \in \mathcal{D}_{\omega}^{a c}(M)$, we define the $\rho$-capacity of $L$ by

$$
\gamma(L):=\rho\left(H, \mu_{M}\right)-\rho(H, 1)
$$

for any $H \mapsto L$.

The following is Theorem III (1) which is the analogoue of Corollary 2.3 in $[\mathrm{V}]$.

Theorem 7.2. We have

$$
\gamma(L)=0 \quad \text { if and only if } L=o_{M} .
$$

One can also easily derive from Theorem 6.4 and Theorem 7.2 that the Hofer's distance in (2.10) [O4] is nondegenerate. In fact, we have the inequality

$$
\gamma(L) \leq d\left(L, o_{M}\right)
$$

since we have

$$
\rho\left(H, \mu_{M}\right) \leq \int_{0}^{1}-\min H_{t} d t \quad \text { and } \rho(H, 1) \geq \int_{0}^{1}-\max H_{t} d t
$$

by Theorem 6.4 (1).

Using $\gamma$ applied to the compactification of the graph of Hamiltonian diffeomorphisms on $\mathbb{R}^{2 n}$ as in [V], one can define an invariant $\gamma(\phi)$ of Hamiltonian diffeomorphisms $\phi$ of $\mathbb{R}^{2 n}$. Then (7.1) has the implication

$$
\gamma(\phi) \leq\|\phi\|=\text { Hofer's norm of } \phi
$$

which is the analogoue to Corollary 1.2.C. [BP] i.e, the inequality between Hofer's and Viterbo's norm. Here we prove this inequality directly without proving the local flatness. 
The rest of this section will be spent to prove Theorem 7.2. It turns out easier to work with the geometric version of the Floer homology to prove Theorem 7.2. We consider the equation

$$
\left\{\begin{array}{l}
\frac{\partial u}{\partial \tau}+J \frac{\partial u}{\partial t}=0 \\
u(\tau, 0) \in \phi_{H}\left(o_{M}\right), u(\tau, 1) \in o_{M}
\end{array}\right.
$$

which is the gradient flow of $\underline{a}_{H}$ normalized as in Section 8 [O4] so that $\mathcal{A}_{H}\left(z_{H}^{p}\right)=\underline{a}_{H}(p)$ for all $p \in \phi_{H}\left(o_{M}\right) \cap o_{M}$. Then we have $\widetilde{\rho}(H, u)=\rho(H, u)$ for all $u \in H^{*}(M, \mathbb{Z})$ and in particular

$$
\widetilde{\rho}\left(H, \mu_{M}\right)-\widetilde{\rho}(H, 1)=\rho\left(H, \mu_{M}\right)-\rho(H, 1) .
$$

Therefore to prove the theorem, it will be enough to prove

$$
\widetilde{\rho}\left(H, \mu_{M}\right)-\widetilde{\rho}(H, 1)>0
$$

when $\phi_{H}\left(o_{M}\right) \neq o_{M}$. Let $\phi_{H}\left(o_{M}\right) \neq o_{M}$ and define

$$
d_{H}:=\max \left\{|p|_{g} \mid p \in \phi_{H}\left(o_{M}\right) \subset T^{*} M\right\}
$$

which then will be strictly positive. Here $|p|_{g}$ denotes the induced norm of $p \in T_{\pi(p)}^{*} M$ with respect to the metric $g$ on $M$ (as a linear functional on $\left.T_{\pi(p)} M\right)$.

By making a $C^{1}$-small perturbation of $H$ into $H^{\prime}$, we may assume

$$
d_{H^{\prime}}>d_{H}-\epsilon>0
$$

and

$$
\left|\widetilde{\rho}\left(H^{\prime}, u\right)-\widetilde{\rho}(H, u)\right| \leq \epsilon
$$

for all $u \in H^{*}(M, \mathbb{Z})$, where $\epsilon$ can be made arbitrarily small. In fact, (7.5) follows from Theorem 6.4 (2). The following lemma can be proven by a standard argument by contradiction.

Lemma 7.3. Denote by $B_{\delta_{0}}\left(p_{0}\right)$ the $\delta_{0}$-ball centered at $p_{0}$ in $T^{*} M$ where $p_{0} \in \phi_{H}\left(o_{M}\right)$ is a point with $\left|p_{0}\right|=d_{H}$. Then there exists constants $c=$ $c(H, J)$ and $\epsilon_{0}>0$ such that for any $H^{\prime}$ with $\left\|H^{\prime}-H\right\|_{C^{1}}<\epsilon_{0}$, we have

$$
\mathcal{A}_{H^{\prime}}(u(-\infty))-\mathcal{A}_{H^{\prime}}(u(\infty)) \geq c>0
$$


for any solution $u$ of

$$
\left\{\begin{array}{l}
\frac{\partial u}{\partial \tau}+J \frac{\partial u}{\partial t}=0 \\
u(\tau, 0) \in \phi_{H^{\prime}}\left(o_{M}\right), u(\tau, 1) \in o_{M}
\end{array}\right.
$$

such that

$$
u(0,0) \in B_{\delta_{0}}\left(p_{0}\right) \cap \phi_{H^{\prime}}\left(o_{M}\right) .
$$

We now recall the definition of the cap action by $\mu_{M}$ on $H F_{*}\left(\phi_{H^{\prime}}\left(o_{M}\right), o_{M}\right)$ (see [BzR] or [PSS]). This action is the dual version of the cup action $(\cdot) \cup \mu_{M}$ which we define in Section 5: Pick a generic point $p \in \phi_{H^{\prime}}\left(o_{M}\right)$ and count the number, denoted by $n_{\{p\}}\left(z^{\alpha}, z^{\beta}\right)$ of solutions of (7.7) that passes through the point $p$ via the evaluation map from $\mathcal{M}_{J}\left(z^{\alpha}, z^{\beta}\right)$. Because of the dimensional reason, we could have $n_{\{p\}}\left(z^{\alpha}, z^{\beta}\right) \neq 0$ only when

$$
\mu\left(z^{\alpha}\right)-\mu\left(z^{\beta}\right)=n
$$

Then we consider the assignment

$$
z^{\alpha} \mapsto \sum_{z^{\alpha}, z^{\beta}} n_{\{p\}}\left(z^{\alpha}, z^{\beta}\right) z^{\beta}
$$

and extend linearly to $C F_{*}\left(\phi_{H^{\prime}}\left(o_{M}\right), o_{M}\right)$. The cap action by $\mu_{M}$ on

$$
H F_{n}\left(\phi_{H^{\prime}}\left(o_{M}\right), o_{M}\right)
$$

is then defined by

$$
\mu_{M} \cap_{F}\left[z^{\alpha}\right]=\sum_{z^{\alpha}, z^{\beta}} n_{\{p\}}\left(z^{\alpha}, z^{\beta}\right)\left[z^{\beta}\right]
$$

for $z^{\alpha} \in C F_{n}\left(\phi_{H^{\prime}}\left(o_{M}\right), o_{M}\right)$ and by proving that it descends to

$$
H F_{n}\left(\phi_{H^{\prime}}\left(o_{M}\right), o_{M}\right)
$$

The following lemma can be proven by a straightforward modification of the main theorem of [F3]. We refer to [PSS] or [BzR] for further explanation on the cap action. 
Lemma 7.4. Let $K$ be a generic choice of Hamiltonian. Consider the cap action by $\mu_{M} \in H^{n}(M, \mathbb{Z})$ on $H F_{n}\left(\phi_{K}\left(o_{M}\right), o_{M}\right)$

$$
\mu_{M} \cap_{F}(\cdot): H F_{n}\left(\phi_{K}\left(o_{M}\right), o_{M}\right) \rightarrow H F_{0}\left(\phi_{K}\left(o_{M}\right), o_{M}\right) .
$$

This action becomes an isomorphism with $\mathbb{Z}$-coefficients (resp. $\mathbb{Z}_{2}$-coefficients) when $M$ is orientable (resp. when $M$ is non-orientable) and restricts to a homomorphism

$$
\mu_{M} \cap_{F}(\cdot): H F_{n}^{(-\infty, \lambda)}\left(\phi_{K}\left(o_{M}\right), o_{M}\right) \rightarrow H F_{0}^{(-\infty, \lambda)}\left(\phi_{K}\left(o_{M}\right), o_{M}\right)
$$

for any $\lambda \in \mathbb{R}$.

To prove Theorem 7.2, we need to refine (7.9), i.e, we need to decrease the filtration level by a positive amount. Let $a \in C F_{n}\left(\phi_{H^{\prime}}\left(o_{M}\right), o_{M}\right)$ and $b \in$ $C F_{0}\left(\phi_{H^{\prime}}\left(o_{M}\right), o_{M}\right)$ whose Floer homology class $\left.[a] \in H F_{n}\left(\phi_{H^{\prime}}\left(o_{M}\right), o_{M}\right)\right)$ and $[b] \in H F_{0}\left(\phi_{H^{\prime}}\left(o_{M}\right), o_{M}\right)$ become the generators respectively. Then we write

$$
\begin{aligned}
& {[a]=\left[\sum_{\ell} a_{\ell} z_{\ell}\right]} \\
& {[b]=\left[\sum_{k} a_{k} z_{k}\right]}
\end{aligned}
$$

where $z_{\ell}, z_{k} \in \operatorname{Crit}\left(\underline{a}_{H}\right)$ and we assume that $\left[z_{\ell}\right] \neq 0 \neq\left[z_{k}\right]$. We define

$$
\begin{aligned}
& C_{a}:=\left\{z_{\ell} \in \operatorname{Crit}\left(\underline{a}_{H}\right) \mid a_{\ell} \neq 0\right\} \\
& C_{b}:=\left\{z_{k} \in \operatorname{Crit}\left(\underline{a}_{H}\right) \mid a_{k} \neq 0\right\}
\end{aligned}
$$

and the subset of $\phi_{H^{\prime}}\left(o_{M}\right)$

$$
N_{a b}:=\left\{p \in \phi_{H^{\prime}}\left(o_{M}\right) \mid p=u(0,0) \text { for some } u \in \bigcup_{\left(z_{\ell}, z_{k}\right) \in C_{\ell} \times C_{k}} \mathcal{M}_{J}\left(z_{\ell}, z_{k}\right)\right\} .
$$

Then from the definition of the cap action, the first statement of Lemma 7.4 implies

$$
\overline{N_{a b}}=\phi_{H^{\prime}}\left(o_{M}\right) .
$$

(See [F3]). Otherwise it is easy to see by choosing a generic point in $\phi_{H^{\prime}}\left(o_{M}\right) \backslash \overline{N_{a b}}$ that the cap action will be trivial. If we choose the point $p$ from $B_{\delta_{0}}\left(p_{0}\right) \cap \phi_{H^{\prime}}\left(o_{M}\right)$, we have

$$
\mathcal{A}_{H^{\prime}}(u(-\infty))-\mathcal{A}_{H^{\prime}}(u(+\infty)) \geq c
$$


by Lemma 7.3. Therefore it follows that the cap action induces a homomorphism

$$
\mu_{M} \cap_{F}(\cdot): H F_{n}^{(-\infty, \lambda)}\left(\phi_{H^{\prime}}\left(o_{M}\right), o_{M}\right) \rightarrow H F_{0}^{(-\infty, \lambda-c)}\left(\phi_{H^{\prime}}\left(o_{M}\right), o_{M}\right)
$$

for any $\lambda \in \mathbb{R}$.

Proof of Theorem 7.2. Suppose the contrary, i.e., assume that

$$
\rho\left(H, \mu_{M}\right)-\rho(H, 1)=0 .
$$

We choose a generic $H^{\prime}$ that satisfy (7.6) and Lemma 7.3, we have

$$
0 \leq \rho\left(H^{\prime}, \mu_{M}\right)-\rho\left(H^{\prime}, 1\right)<\epsilon<\frac{c}{2} .
$$

We now consider the following commutative diagram

$$
\begin{array}{ccc}
H F_{0}^{(-\infty, \lambda-c)}\left(\phi_{H^{\prime}}\left(o_{M}\right), o_{M}\right) \stackrel{j_{*}^{(\lambda-c)}}{\longrightarrow} & H F_{0}\left(\phi_{H^{\prime}}\left(o_{M}\right), o_{M}\right) \\
\uparrow \mu_{M} \cap_{F}(\cdot) & \uparrow \mu_{M} \cap_{F}(\cdot) \\
H F_{n}^{(-\infty, \lambda)}\left(\phi_{H^{\prime}}\left(o_{M}\right), o_{M}\right) & \stackrel{j_{*}^{\lambda}}{\longrightarrow} & H F_{n}\left(\phi_{H^{\prime}}\left(o_{M}\right), o_{M}\right)
\end{array}
$$

The right vertical arrow is an isomorphism by (7.9). From this, we see that whenever the bottom arrow becomes non-zero, the top arrow becomes non-zero. However $\widetilde{F}_{H^{\prime}}(1)$ or $\widetilde{F}_{H^{\prime}}\left(\mu_{M}\right)$ is the generator of $H F^{0}$ or $H F^{n}$ respectively by the Floer-Thom isomorphism and it is easy to check that the non-zeroness of these homomorphisms are equivalent to $j_{\lambda}^{*} \widetilde{F}_{H^{\prime}}(1) \neq 0$ or $j_{\lambda-c}^{*} \widetilde{F}_{H^{\prime}}\left(\mu_{M}\right) \neq 0$ respectively. Here we denote by $\widetilde{F}_{H}$ the Floer-Thom isomorphism for the geometric version of the Floer cohomology

$$
\widetilde{F}_{H}: H^{*}(M, \mathbb{Z}) \rightarrow H F^{*}\left(\phi_{H^{\prime}}\left(o_{M}\right), o_{M}\right) .
$$

Combining these, we have derived

$$
\rho\left(H^{\prime}, \mu_{M}\right) \geq \rho\left(H^{\prime}, 1\right)+c .
$$

This gives rise to a contradiction to (7.13). Therefore the result follows. 


\section{Appendix: Index computation on $\Theta_{0,1}$.}

First, note that the operator glued in the cap in the proof of Theorem 3.3 is of the following type: Consider the domain $\Theta_{0,1} \subset \mathbb{C}$ as drawn in the picture which is conformally a disc with one marked point.

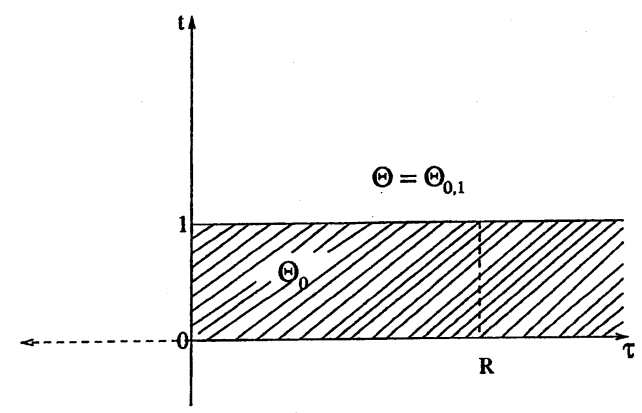

Figure 9

We consider the operator

$$
\bar{\partial}_{J, T}=\frac{\partial}{\partial \tau}+J \frac{\partial}{\partial t}+T: \quad W_{n}^{1, p}\left(\Theta_{0,1}\right) \rightarrow L^{p}\left(\Theta_{0,1}\right), \quad p>2
$$

on the space

$$
W_{n}^{1, p}=W_{n}^{1, p}\left(\Theta_{0,1}\right):=\left\{\zeta \in W^{1, p}\left(\Theta_{0,1}, \mathbb{C}^{n}\right) \mid \zeta(\theta) \in \mathbb{R}^{n} \text { for all } \theta \in \partial \Theta_{0,1}\right\}
$$

where $J$ and $T$ satisfy (1)-(4) in Section 5 [O4] as $\tau \rightarrow \infty$. Such an operator $\bar{\partial}_{J, T}$ is a Fredholm operator and so has the Fredholm index. The following index formula is the main theorem we will prove in this appendix.

Theorem A.1. Let $T$ and $J$ be as above and $\Psi_{\infty}$ be the solution of

$$
\left\{\begin{array}{l}
\frac{\partial \Psi}{\partial t}-J(\infty, t) T(\infty, t) \Psi=0 \\
\Psi(0)=i d
\end{array}\right.
$$

Then we have

$$
\operatorname{Index} \bar{\partial}_{J, T}=\mu\left(\Psi_{\infty}(t) \cdot \mathbb{R}^{n}, \mathbb{R}^{n}\right)+\frac{n}{2} .
$$

One immediate corollary is the following index formula. This can be proved by considering the linearized operator which will have the form of the operator considered as in Theorem A.1 under the trivialization of the type we used in Section 5.1 [O4]. 
Corollary A.2. Let $R>0$ be a real number. Consider the perturbed Cauchy-Riemann equation of the map $u: \Theta_{0,1} \rightarrow T^{*} M$

$$
\left\{\begin{array}{l}
\frac{\partial u}{\partial \tau}+J\left(\frac{\partial u}{\partial t}-X_{H}(u)\right)=0, \quad \text { on } \tau>R+1 \\
u \quad \text { satisfying appropriate zero-order perturbation } \\
\quad \text { of the equation } \frac{\partial u}{\partial \tau}+J \frac{\partial u}{\partial t}=0, \quad \text { for } \tau \leq R+1
\end{array}\right.
$$

Then if the Hamiltonian path $z(t)=z_{H}^{p}(t)$ for $p \in \phi_{H}\left(o_{M}\right) \cap o_{M}$ is regular (i.e., $\phi_{H}\left(o_{M}\right) \pitchfork o_{M}$ at $p$ ) and so the linearization at $u$ becomes a Fredholm operator, then we have

$$
\text { Index } u=-\mu(z)+\frac{n}{2} .
$$

The remaining section will be spent to prove Theorem A.1.

By deforming the operators without changing the Fredholm index, we may further assume that for sufficiently large $R>0$

$$
J(\tau, \cdot)=\left\{\begin{array}{lll}
J(\infty, \cdot) & \text { for } & \tau \geq R+1 \\
i & \text { for } & \tau \leq R
\end{array}\right.
$$

and

$$
T(\tau, \cdot)=\left\{\begin{array}{lll}
T(\infty, \cdot) & \text { for } & \tau \geq R+1 \\
0 & \text { for } \quad \tau \leq R
\end{array}\right.
$$

We now transform the above operator on $W_{n}^{1, p}$ to another operator defined on the space

$$
W_{\widetilde{\Lambda}}^{1, p}=\left\{\zeta \in W^{1, p}\left(\Theta_{0,1}, \mathbb{C}^{n}\right) \mid \zeta(\theta) \in \widetilde{\Lambda}(\theta)\right\}
$$

where $\widetilde{\Lambda}: \partial \Theta_{0,1} \rightarrow \widetilde{\Lambda}(n)$ is the path defined as

$$
\widetilde{\Lambda}(\theta)=\left\{\begin{array}{lll}
\mathbb{R}^{n} & \text { if } \quad \theta \in \partial_{0} \Theta_{0,1} \\
\mathbb{R}^{n} & \text { if } \quad \theta=(\tau, 0) \text { for } \tau \leq R+1 \\
\widetilde{\Lambda}_{1}(\theta) & \text { if } \quad \theta=(\tau, 1) \text { for } R+1 \leq \tau \leq R+2 \\
\Psi(1) \cdot \mathbb{R}^{n} & \text { if } \quad \theta=(\tau, 1) \text { for } \tau \geq R+2 .
\end{array}\right.
$$

Here $\partial_{0} \Theta_{0,1}$ is the portion of the boundary of the compact part $\Theta_{0}$ in $\partial \Theta_{0,1}$ as pictured in Figure 9 and $\widetilde{\Lambda}_{1}:[R+1, R+2] \rightarrow \widetilde{\Lambda}(n)$ is a path defined by

$$
\widetilde{\Lambda}_{1}(\tau, 1):=\Psi_{\infty}(\rho(\tau-R-1)) \cdot \mathbb{R}^{n} \quad \text { for } \quad \tau \geq R-1
$$


and by extending this definition to the whole $\Theta$ by setting

$$
\widetilde{\Lambda}(\theta) \equiv \mathbb{R}^{n} \quad \text { for } \quad \tau \leq R-1 .
$$

Here the function $\rho: \mathbb{R} \rightarrow[0,1]$ is the cut-off function we used before in the main part of this paper.

Note that since we assume that $T \equiv 0$ on $\tau \leq R$, the map $\Psi:[R, \infty) \times$ $[0,1] \rightarrow S p(2 n)$ defined by

$$
\Psi(\tau, t):=\Psi(\rho(\tau-R-1) t) \quad \text { for } \quad \tau \geq R
$$

can be smoothly extended to the whole $\Theta$ by setting $\Psi \equiv$ id for $\tau \leq R$. Therefore we can now define the push-forward operator

$$
\Psi_{*}\left(\bar{\partial}_{J, T}\right)=\Psi \circ \bar{\partial}_{J, T} \circ \Psi^{-1}: \quad W_{\bar{\chi}}^{1, p} \rightarrow L^{p} .
$$

Then this push-forward will have the form

$$
\bar{\partial}_{\mathcal{J}, \widetilde{T}, \Lambda}=\frac{\partial}{\partial \tau}+\widetilde{J} \frac{\partial}{\partial t}+\widetilde{T}: \quad W_{\Lambda}^{1, p} \rightarrow L^{p}
$$

such that

$$
\begin{aligned}
\widetilde{T} \equiv 0 & \text { if } \tau \geq R+1 \quad \text { or } \quad \tau \leq R \\
\widetilde{J} \equiv i & \text { if } \tau \leq R
\end{aligned}
$$

It is obvious that the two operators $\bar{\partial}_{J, T}$ and $\bar{\partial}_{J_{,}, \widetilde{T}, \Lambda}$ will have the same Fredholm indices and so it is enough to compute the index of $\bar{\partial}_{\jmath, \widetilde{T}, \Lambda}$. We recall that we imposed the transversality $\Psi(1) \cdot \mathbb{R}^{n} \pitchfork \mathbb{R}^{n}$ and so

$$
\widetilde{\Lambda}_{1}(\theta) \pitchfork \mathbb{R}^{n} \quad \text { for } \quad \theta=(\tau, 1), \quad \tau \geq R+2
$$

If we denote

$$
\infty_{+}=\lim _{\tau \rightarrow \infty}(\tau, 1) \quad \text { and } \infty_{-}=\lim _{\tau \rightarrow \infty}(\tau, 0)
$$

the map $\widetilde{\Lambda}: \partial \Theta_{0,1} \rightarrow \widetilde{\Lambda}(n)$ satisfies

$$
\begin{aligned}
\widetilde{\Lambda}\left(\infty_{-}\right) & =\widetilde{\Lambda}(\tau, R+1)=\mathbb{R}^{n} \quad \text { and } \quad \widetilde{\Lambda}\left(\infty_{+}\right)=\Psi(1) \cdot \mathbb{R}^{n}=\widetilde{\Lambda}_{1}(R+1) \\
\widetilde{\Lambda}(\tau, 1) & =\Psi(\rho(\tau-R-1)) \cdot \mathbb{R}^{n} \quad \text { for } \quad R+1 \leq \tau .
\end{aligned}
$$

By construction, the two paths $t \mapsto \Psi(t) \cdot \mathbb{R}^{n}$ and $\tau \mapsto \widetilde{\Lambda}_{1}$ are homotopic with the same fixed end points and so have the same Maslov indices, i.e.,

$$
\mu\left(\widetilde{\Lambda}_{1}, \mathbb{R}^{n}\right)=\mu\left(\Psi \cdot \mathbb{R}^{n}, \mathbb{R}^{n}\right) .
$$


Using the stratum-homotopy invariance of the Maslov index, Theorem 2.4 [RS1], we can deform the operator $\bar{\partial}_{J, \widetilde{T}, X}$ without changing the Fredholm property and without changing the Maslov indices of $\widetilde{\Lambda}$ into

$$
\bar{\partial}_{i, 0, \Lambda}=\frac{\partial}{\partial \tau}+i \frac{\partial}{\partial t}=\bar{\partial}: \quad W_{\Lambda}^{1, p} \rightarrow L^{p}
$$

where $\Lambda: \partial \Theta_{0,1} \rightarrow \Lambda(n)$ is defined as

$$
\Lambda(\theta)=\left\{\begin{array}{lll}
\mathbb{R}^{n} & \text { if } & \theta=(\tau, 0) \\
D(t) \cdot \mathbb{R}^{n} & \text { if } & \theta=(t, 0) \\
i \cdot \mathbb{R}^{n} & \text { if } & \theta=(\tau, 1)
\end{array}\right.
$$

with

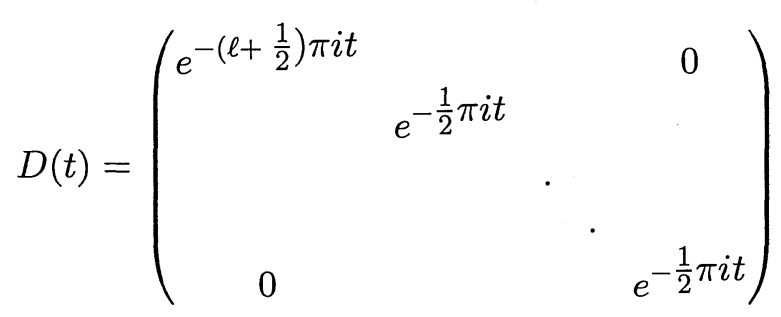

for some integer $\ell$. Here we identify $\Theta_{0,1}$ with the semi-strip as drawn below.

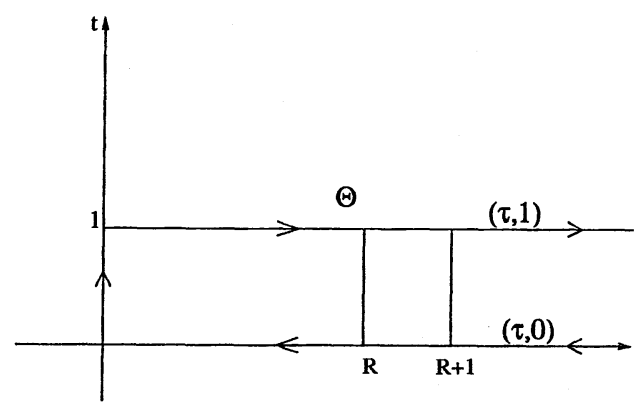

Figure 10

Noting that both the operator $\bar{\partial}$ and the boundary condition $\Lambda$ both are separable, we have reduced the computation of the index into the onedimensional problem below. Recall that both Fredholm index of $\bar{\partial}_{i, 0, \Lambda}$ and the Maslov index of $\Lambda$ are additive under the direct sum.

We now study the following equation in one-dimension

$$
\left\{\begin{array}{l}
\bar{\partial} \zeta=0 \\
\zeta(\tau, 0) \in \mathbb{R}, \quad \zeta(\tau, 1) \in i \mathbb{R} \\
\zeta(0, t) \in e^{-\left(\ell+\frac{1}{2}\right) \pi i t} \mathbb{R}
\end{array}\right.
$$


Note that $\zeta$ satisfying (A.2), (A.3) and $\int|D \zeta|^{2}<\infty$ must have the form

$$
\zeta(z)=\sum_{k=1}^{\infty} a_{k} e^{-\left(k-\frac{1}{2}\right) \pi z}, \quad a_{k} \in \mathbb{R} \quad \text { and } z \in \Theta_{0,1} .
$$

This becomes

$$
\zeta(0, t)=\sum_{k=1}^{\infty} a_{k} e^{-\left(k-\frac{1}{2}\right) \pi i t}
$$

on $\{0\} \times[0,1]$. Since (A.4) implies

$$
e^{\left(\ell+\frac{1}{2}\right) \pi i t} \zeta(0, t) \in \mathbb{R}
$$

we have

$$
\sum_{k=1}^{\infty} a_{k} e^{-(k-\ell-1) \pi i t} \in \mathbb{R} .
$$

Equivalently, we have

$$
\sum_{k=-\ell}^{\infty} a_{k+\ell+1} e^{-k \pi i t} \in \mathbb{R}
$$

We consider two cases where $\ell \geq 0$ and $\ell \leq-1$ separately. First, let us assume that $\ell \geq 0$. Then from (A.5), we derive

$$
\begin{aligned}
a_{k+\ell+1}=0 & \text { if } k \geq \ell+2 \\
a_{\ell+1} & \text { arbitrary } \\
a_{k+\ell+1}=a_{-k+\ell+1} & \text { if }-\ell \leq k \leq-1
\end{aligned}
$$

i.e.,

$$
\begin{aligned}
a_{k}=0 & \text { if } k \geq \ell+2 \\
a_{\ell+1} & \text { arbitrary } \\
a_{k}=a_{-k+2(\ell+1)} & \text { if } 1 \leq k \leq \ell
\end{aligned}
$$

On the other hand if $\ell \leq-1$, it immediately follows from (A.5) that (*) has no non-trivial solution. Hence if we denote by $\operatorname{Ker}[*]$ the solution space of the equation $(*)$, then we have

$$
\operatorname{dim} \operatorname{Ker}[*]=\left\{\begin{array}{lll}
\ell+1 & \text { if } \quad \ell \geq 0 \\
0 & \text { if } \quad \ell \leq-1
\end{array}\right.
$$


Now let us study the $L^{2}$-adjoint problem of $(*)$;

$(* *) \quad\left\{\begin{array}{l}\partial \eta=0, \\ \eta(\tau, 0) \in \mathbb{R}, \quad \eta(\tau, 1) \in i \mathbb{R} \\ i \eta(0, t) \in e^{-\left(\ell+\frac{1}{2}\right) \pi i t} \cdot \mathbb{R}\end{array}\right.$

and denote by Coker $[*]$ the solution space of $(* *)$. This equation can be derived by taking the $L^{2}$-inner product $(*)$ with $\eta$ and then by integrating by parts. It follows from (A.7), (A.8) and $\int|D \eta|^{2}<\infty$ that $\eta$ must have the form

$$
\eta(z)=\sum_{j=1}^{\infty} b_{j} e^{-\left(j-\frac{1}{2}\right) \pi \bar{z}}, \quad b_{j} \in \mathbb{R} .
$$

By substituting $z=(0, t)$ into this, we get

$$
\eta(0, t)=\sum_{j=1}^{\infty} b_{j} e^{\left(j-\frac{1}{2}\right) \pi i t}, \quad b_{j} \in \mathbb{R} .
$$

Condition (A.9) is equivalent to

$$
i e^{\left(\ell+\frac{1}{2}\right) \pi i t} \eta(0, t) \in \mathbb{R} \quad \text { i.e., } \quad \sum_{j=1}^{\infty} i b_{j} e^{(j+\ell) \pi i t} \in \mathbb{R}
$$

and hence we have derived

$$
\sum_{j=\ell+1}^{\infty} i b_{j-\ell} e^{j \pi i t} \in \mathbb{R}
$$

From this, we immediately conclude that if $\ell \geq 0$, then $(* *)$ has no nontrivial solution. When $\ell \leq-1$, we derive from (A.10)

$$
\begin{array}{rll}
b_{j-\ell}=0 & \text { if } \quad j \geq-\ell \\
b_{-\ell}=0 & & \\
b_{j-\ell}=-b_{-j-\ell} & \text { if } \quad 1 \leq j \leq-\ell-1
\end{array}
$$

From this, we conclude that

$$
\operatorname{dim} \operatorname{Coker}[*]=\left\{\begin{array}{lll}
0 & \text { if } \quad \ell \geq 0 \\
-\ell-1 & \text { if } \quad \ell \leq-1
\end{array}\right.
$$


Combining (A.6) and (A.11), we have proven

$$
\operatorname{Index}[*]=\operatorname{dim} \operatorname{Ker}[*]-\operatorname{dim} \operatorname{Coker}[*]=\ell+1
$$

for all $\ell$. By applying (A.12) for $\ell=0$ and adding the contributions from other components of $D(t)$, we get

$$
\operatorname{Index} \bar{\partial}_{i, 0, \Lambda}=\ell+1+(n-1)=\ell+n
$$

We now compute the Maslov index $\mu\left(D(t) \cdot \mathbb{R}^{n}, \mathbb{R}^{n}\right)$ of the path

$$
t \mapsto D(t) \cdot \mathbb{R}^{n} .
$$

By the additivity of the Maslov index under the direct sum operation, we have

$$
\mu\left(D(t) \cdot \mathbb{R}^{n}, \mathbb{R}^{n}\right)=\mu\left(e^{-i\left(\ell+\frac{1}{2}\right) \pi t} \cdot \mathbb{R}, \mathbb{R}\right)+(n-1) \mu\left(e^{-\frac{\pi}{2} i t} \cdot \mathbb{R}, \mathbb{R}\right) .
$$

However, it is easy to check from the definition of the Maslov index from [RS1], we have

$$
\mu\left(e^{-i\left(\ell+\frac{1}{2}\right) \pi t} \cdot \mathbb{R}, \mathbb{R}\right)=\ell+\frac{1}{2} .
$$

By applying this to $\ell=0$ for the second term in (A.14) as well, we conclude that

$$
\mu\left(D \cdot \mathbb{R}^{n}, \mathbb{R}^{n}\right)=\ell+\frac{n}{2} .
$$

However from (A.1) and from the way how we deform the operators afterwards, we have

$$
\mu\left(\Psi \cdot \mathbb{R}^{n}, \mathbb{R}^{n}\right)=\mu\left(D \cdot \mathbb{R}^{n}, \mathbb{R}^{n}\right)
$$

which finally finishes Theorem A.1.

\section{References.}

[BzC] M. Betz and R. Cohen, Graph moduli spaces and cohomology operations, Turkish J. Math. 18 (1995), 23-41.

[BzR] M. Betz and J. Rade, Products and relations in symplectic Floer homology, preprint. 
[BP] M. Bialy and L. Polterovich, Geodesics of Hofer's metric on the group of Hamiltonian diffeomorphisms, Duke Math. J. 76 (1994), 273-292.

[F1] A. Floer, Morse theory for Lagrangian intersections, J. Differ. Geom. 28 (1988), 513-547.

[F2] A. Floer, Witten's complex and infinite dimensional Morse theory, J. Differ. Geom. 30 (1989), 207-221.

[F3] A. Floer, Cup-length estimates for Lagrangian intersections, Comm. Pure Appl. Math. 42 (1989), 335-356.

[FH1] A. Floer and H. Hofer, Coherent orientations for periodic orbit problems in symplectic geometry, Math. Z. 212 (1993), 13-38.

[FH2] A. Floer and H. Hofer, Symplectic homology I: Open sets in $\mathbb{C}^{n}$, Math. Z. 215 (1994), 37-88.

[Fu1] K. Fukaya, Morse homotopy and its quantization, AMS/IP Studies in Advanced Math., 2 (1997), 409 - 440.

[Fu2] K. Fukaya, Morse theory and topological field theory, Sugaku Expositions, 10, No 1 (1997), 19-39.

[FO] K. Fukaya and Y.-G. Oh, Zero-loop open strings in the cotangent bundle and Morse homotopy, Asian J. Math. 1 (1997), 96-180.

[Mi] J. Milnor, Lectures on the h-Cobordism Theorem, Princeton University Press, Princeton, New Jersey, 1965.

[Mk] D. Milinković, Thesis in the University of Wisconsin-Madison, 1997.

[MO] D. Milinković and Y.-G. Oh, Generating functions versus action functional, CRM Proceedings and Lecture Notes, Vol. 15, 107-125, eds. by F. Lalonde, 1998, AMS.

[O1] Y.-G. Oh, Floer cohomology of Lagrangian intersections and pseudoholomorphic disks I, Comm. Pure Appl. Math. 46 (1993), 949-994.

[O2] Y.-G. Oh, Fredholm theory of holomorphic discs under the perturbation of boundary condition, Math. Z. 222 (1996), 505-520.

[O3] Y.-G. Oh, Relative Floer and quantum cohomology and the symplectic topology of Lagrangian submanifolds, Publ. Newton. Inst. 8, 201-267, eds. by C. B. Thomas, Cambridge University Press, 1996, Cambridge, England.

[O4] Y.-G. Oh, Symplectic topology as the geometry of action functional, I, J. Differ. Geom. bf 46 (1997), 499-577. 
[O5] Y.-G. Oh, Floer cohomology, spectral sequence and the Maslov class of Lagrangian embeddings, Intern. Math. Res. Notices, 7 (1996), 305-340.

[O6] Y.-G. Oh, Gromov-Floer theory and disjunction energy of compact Lagrangian embeddings, Math. Res. Let. 4 (1997), 895-905.

[PSS] S. Piunikhin, D. Salamon and M. Schwarz, Symplectic Floer-Donaldson theory and quantum cohomology, Publ. Newton. Inst. 8, 171-200, eds. by C. B. Thomas, Cambridge University Press, 1996, Cambridge, England.

[RS] J. Robbin and D. Salamon, The Maslov index for paths, Topology, 32 (1993), $827-844$.

[RT] Y. Ruan and G. Tian, Bott-type symplectic Floer cohomology and its multiplication structures, Math. Res. Let. 2 (1995), 203-219.

[Sc1] M. Schwarz, Morse Homology, Progress in Math. 111, Birkhäuser, 1993.

[Sc2] M. Schwarz, Thesis in ETH, Zürich, 1995.

[V] C. Viterbo, Symplectic topology as the geometry of generating functions, Math. Ann. 292 (1992), 685-710.

RECEIVED MAY 9, 1996 AND REVISED MAY 23, 1997.

Korea Institute For Advanced Study

207-43 ChUNGRYANGRI-DONG

DongdaEmun-KU, SEOUl 130-02

E-MAIL: YOH@KIAS.RE.KR

AND

Department of MATHEMATICS

UNIVERSITY OF WISCONSIN

MADISON, WI 53706

E-MAIL: OH@MATH.WISC.EDU 